\title{
A Three-Tiered Theory of the Human Conceptual System Jianqiu Zhang michelle.zhang@utsa.edu
}

\section{Introduction}

Concepts are the building blocks of reasoning, decision making, and other cognitive tasks. The human conceptual system is the basic tool utilized in all areas of scientific research.

However, considering the importance of the human conceptual system, little is known about its organizational and operational principles, despite decades of research in philosophy, psychology, and artificial intelligence. For many years, concepts have been understood as stand-alone entities that bundle sets of features and knowledge in the long-term memory. The debate has been whether their contents are definitions, prototypes, exemplars or theories (Rosch and Mervis 1975; Hampton 1979; Brooks 1978; Medin and Schaffer 2017; Murphy and Medin 1985; Carey 1985). In all these theories, concepts are predicated on some form of description (Laurence and Margolis 1999) .

Theories that define concepts as descriptions of real world objects cannot explain why wrong descriptions can be associated with concepts at their inception, yet it is still possible to modify these descriptions later without changing their identity. For example, whales were first described as fish although they have been described as mammals later. This change of the description has not changed the identity of the concept of whales. Consequently, concepts' descriptions cannot be equated to their identity. To overcome such problems, concepts have been further perceived as "pointers" to real objects (Putnam 1975; Kripkie 1980) so that their referents will not be affected when their descriptions change. Furthermore, dual-process theorists posit that features associated with a concept could serve different purposes in various cognitive processes. In particular, there should be one set of features for the identification of concept referents and another set for concept description (Block and Campell 1987; Macnamara 1986; Laurence and Margolis 2002).

Besides opposing views on concept content, a closer examination from both philosophical (Fodor 1975, 1981) and acquisitional perspectives (Carey 2000), reveals the existence of primitive/atomic concepts that cannot be defined by other concepts. Some primitive/atomic concepts have been attributed to evolution-endowed innate analyzers (Laurence and Margolis 2015; Carey 2000) shared across multiple species. While it is true that most concepts in the dictionary are not definable as atomists argue (Fodor 1981) it is also hard to convince the majority of scholars that all these primitive concepts are innate concepts to be discovered without the need for a learning process (Prinz 2005). In addition to these primitive concepts, we also have not fully accounted for composite, counterfactual and abstract concepts that are formed by combining primitive concepts. 
The structural and functional characterizations of the conceptual system have also been diversified. The default single process narrative has been replaced by multiple process narratives, among which, the two-tiered theory of cognition has been most widely accepted (Stanovich and West 2000; Kahneman 2011; Sloman 1996; Evans and Frankish 2009; Evans 2003). The two-tiered theory describes an evolutionarily-conserved pre-linguistic Conceptual System 1 (CS1) and a post linguistic Conceptual System 2 (CS2). CS1 is characterized as a fast intuitive reasoning system that utilizes concept prototypes and their associations stored in the associative memory. It is an error-prone automatic process without much involvement from the linguistic system. CS2 is a slower and less error-prone system that conducts logical reasoning. Since both CS1 and CS2 relies on formed concepts, an important drawback of this two-tiered theory is that it cannot explain how reasoning is possible in new environments when concept prototypes and concept associations have not been formed in the memory.

The research on concepts is best examined within a system framework in order to derive a coherent theory. Otherwise, the discussion will always result in dialectical arguments like those about nativism vs. empiricism (Laurence and Margolis 2015) or contextualism vs. invariantism (Machery 2015). In this paper, I argue that various concept content forms and system functions suggest the existence of a complex conceptual system constructed from multiple components linked in a specific structure. Different phases of its complex operations result in the fragmented theories that we have observed. The dichotomous tension so frequently seen is characteristic of the first two phases of the thesis->antithesis->synthesis process of theory development described by Hegel (Hegel 1929). In other words, a synthesized conceptual system structure must exist that manifests itself simultaneously as both contextual and invariant, native and empirical. In this paper, I employ a systematic approach to show how these dichotomous phenomena are formed and how various descriptive concept contents can be linked to a three-tiered system structure.

This approach treats the conceptual system as a dynamic system consisting of various functional modules. I first delineated that its input unit must be episodic events, which explains why concept meanings are contextual (Barsalou 1987, 1999; Smith and Samuelson 1997; Malt 2010) and why events are represented in a structured way that is closely tied to causes and goals (Zacks and Tversky 2001). Then I discussed the role of innate analyzers; given that they are dedicated special purpose feature extractors (Brannon 2002; Brannon, Abbott, and Lutz 2004; Brannon and Terrace 1998; McCrink and Wynn 2007; F. Xu and Spelke 2000; Fei Xu, Spelke, and Goddard 2005; Chang and Tsao 2017) I concluded that primitive conceptual nodes are formed through the abstraction and segmentation of innate analyzer outputs. This theory resolves the issues facing massive atomism argued by Fodor, who rightfully observed that most lexical items cannot be defined; yet, it seems implausible that these concepts are all innate. Furthermore, it is recognized that primitive conceptual nodes act as indices for retrieving relevant events to enable event-based reasoning. Based on this insight, I proposed a threetiered structure that added the event-based reasoning system, CSO, as the foundation to CS1 and CS2. This three-tiered theory addresses the important question of how it is possible to conduct reasoning before concept prototypes and associative links are formed in CS1. 
Subsequently, I identified the major functional modules of CS0, CS1, and CS2. Particularly, there exists a Dynamic Event Composition Module (DECM) that takes activated conceptual nodes as inputs and outputs composed events as the basis for CS1 reasoning. This account explains the CS1 characteristics documented in Thinking Fast and Slow (Kahneman 2011). Furthermore, I identified data types likes knowledge systems, variables, pointers, and linked lists, which are crucial for logical reasoning. These data types can be constructed because CS2 conceptual nodes cost little to create and they can be linked together with great flexibility.

Besides the system structure, I have also developed a theory on how the various descriptive concept contents are tied into the system structure: exemplars are embedded in the episodic events at the CSO level; concept prototypes and their associative links are generated at the CS1 level and are then taken up as inputs to the DECM module. Theories of concepts are stored in the CS2 level for logical reasoning.

Because functions of different levels often assume different characteristics, past research that did not differentiate the three tiers often led to conflated and confusing arguments. In this paper, I discussed major conceptual system functions tier by tier, which has resolved some of the dialectical arguments. The major insights are:

1. CSO event-based reasoning is employed by prelinguistic infants and animals. It is the foundation of a three-tiered system structure. Primitive conceptual nodes are generated based on innate analyzer outputs. They derive their meaning from their relationship to the objects in the environment.

2. CS2 conceptual nodes are linked to other conceptual nodes and derive their meaning from their relationship with other concepts.

3. CSO, CS1, and CS2 gradually mature as a child grows and this process explains the discontinuity in concept learning observed in false-belief tasks.

4. CS0, CS1, and CS2 function collaboratively in the human reasoning system.

5. Logical reasoning depends on data types like variables and linked lists that can be constructed with flexibility in CS2.

6. Logical reasoning is constrained by the limitations introduced by the abstraction and segmentation steps during the concept forming process.

7. Concept meaning lies in the set of engaged features of concepts in events, and the concept composition process simulates how the engaged concept features can fit into each other. Such simulation can be based on past events or concept prototypes subjected to the check of logical reasoning.

The discussion in this paper is organized from several perspectives: The conceptual system structure perspective, the conceptual node structure perspective, the reasoning perspective, the evolutionary and developmental perspective, the concept composition perspective, and the subjective and objective perspective.

While there are still many details to work out, I believe that my comprehensive description is a step forward to the systematic understanding of the conceptual system. 


\section{Section 1 Conceptual system structure}

In an attempt to construct a coherent conceptual system theory, the first set of questions ought to be: "What are the inputs to the conceptual system?" "What are the major components and how are they connected?" Surprisingly, there has been little work addressing this aspect. It is widely accepted that a pre-linguistic conceptual system CS1 exists, and a post-linguistic CS2, for which past research has identified many characteristics and functions (Stanovich and West 2000; Kahneman 2011; Sloman 1996; Evans and Frankish 2009; Evans 2003). Yet, from a structural perspective, I have not come across any work that actively seeks to identify major function modules and their operations. From the field of artificial intelligence, deep neural networks can be considered as a conceptual system module (LeCun, Bengio, and Hinton 2015). Even though the most advanced implementation (Devlin et al. 2018; Suraperwata and Suyanto 2020) can perform object identification and natural language translation with great accuracy, these networks are still far from having reasoning capabilities. In the field of cognitive psychology, components such as the innate analyzers have been identified and analyzed (Carey 1999; Gopnik et al. 2004; Carey and Spelke 1994; Michotte 1946; Pearl 2000). However, many important functions of the conceptual system have not been accounted for: both CS1 and CS2 rely on concept prototypes and concept associations, without which, it is not possible to reason associatively in CS1 or logically in CS2. The unanswered question is how can reasoning be conducted in new environments before the establishment of concept prototypes and associative links? In addition, there exists a set CS1 characteristics that has not been accounted for. For example, CS1 tends to make up causally coherent stories based on activated concepts but when subjects are primed by an emotionally effective sentence, their judgment of the subsequent sentence can be changed in CS1 (Kahneman 2011). What kind of functional module is responsible for such behaviors?

At the CS2 level, with the addition of the linguistic system, conceptual nodes can be created and linked together with much more flexibility; consequently, they allow the construction of knowledge systems. Furthermore, CS2 conceptual nodes can be aligned with CS0 primitive conceptual nodes in a Quinian bootstrapping process (Quine 1960, 1969, 1977), as explained by Block and Nersessian (Block 1986; Nersessian 1992). However, the question remains: "What data types and functional modules are necessary for conducting logical reasoning? "

Just like the conceptual system, conceptual nodes might also have complex structures. Various theories have linked concepts to prototypes, examples, and theories. Conceptual atomism, on the other hand, takes the view that lexical concepts have no semantic structure and the content of a concept is determined by its relation to the world rather than by how it is related to other concepts (Fodor 1998). In essence, these theories treat concepts as labels that point to their referents and have no descriptive content themselves (Kripkie 1980). A unified view on concept structure is lacking; most theories can explain some behaviors but not all of them. Three of the most puzzling questions have been: "Why does the meaning of concepts always change with contexts?", "Are most concepts learned or innate?", "Do exemplars, prototypes, and theories exist as multiple entities or are they associated with a core?" 
Regarding the first question, Barsalou noticed that contextual independent $(\mathrm{Cl})$ and contextual dependent (CD) contents are associated with each concept (Barsalou 1987,1999; Smith and Samuelson 1997; Malt 2010). The current debate on conceptual change is about whether it is invariant or contextual. Invariantism holds that there is a stable core of features that is always retrieved (Machery 2015) and contextualism thinks the stable core does not exist at all (Casasanto and Lupyan 2015). Neuroscientific evidence shows that when a made-up concept (greebles) was presented verbally by its sound, motion or character properties during the learning phase, the brain region associated with the stimuli would always be activated afterward in a visual-matching task although accessing the stimuli was not required for the task (James and Gauthier 2003). Such findings and those by Hoenig et al. (Hoenig et al. 2008) confirm that the brain regions associated with learning phase features are always activated even if such features are irrelevant to the task on hand. Consequently, the existence of $\mathrm{Cl}$ features is evident. Although these works further illuminated the nature of contextual meaning change, they did not give a mechanistic explanation of why the learning phase features are always invoked regardless of their utility.

Regarding the second question, the debate has been about how big a role the innate analyzers play in concept acquisition and learning. Massive nativism claims that the majority of concepts are acquired and developed through special purpose innate analyzers while empiricists argue that most concepts are learned through innate general purpose cognitive systems (Laurence and Margolis 2015). While results in neurological research (Verhage et al. 2000) have gradually confirmed the nativist arguments, the debate on how concepts are learned and acquired is still unresolved (Margolis and Laurence 2011; Rey 2014; Carey 2014).

Regarding the third question, the research community has realized that there is not a unified theory on concept since evidence has shown that prototypes, exemplars, and theories all exist. Some researchers hypothesized that concepts are heterogeneous and the notion of concept ought to be eliminated (Machery 2009). Yet others proposed pluralism, which treats concepts as aggregations of multiple types of content tied to their atomic cores (Laurence and Margolis 1999).

There are many unanswered questions; only through a thorough understanding of the conceptforming process and the conceptual system structure, can we reconcile the various phenomena observed in concept research. In this paper, I first approach the problem by identifying the major structural components of the conceptual system.

\section{Section1.1 The input to the concept system must be episodic events}

In a systematic approach, we first need to clarify what are the inputs and outputs. However, this issue has not been extensively discussed and stand-alone elements in episodic events have been implicitly considered as the inputs to the conceptual system. In classical theories that treat concepts as definitions, prototypes, exemplars, or theories, concepts have been thought as mostly mental representations of objects. Papafragou (Papafragou 2015) wrote “...models of 
language acquisition generally assume that learners are equipped with a set of concepts for representing events and their components" (Jackendoff 1992; Gleitman 1990). This is to say that concepts of individual components are learned before they are used for event representation. However, this narrative cannot explain why concept meaning varies with context. One recent theory proposed that there is no memorized mapping between word forms and concepts. Contextual meaning of words is determined by the specifics of the internal neurocognitive and external physical and social contexts when they are retrieved (Casasanto and Lupyan 2015). However, this theory ignores the fact that the stability of meaning increases with the number of words. While words taken out of context can be misunderstood easily, words embedded in context do not. Otherwise, scientific theories or instruction manuals with stable meanings would be impossible. This points to the possibility that the inputs to the conceptual system are actually episodic events. For example, the conceptual system actually takes the whole episode of a dog playing with a kid rather than the dog in isolation as its input. Contextual information embedded in episodic events will influence the set of features we think about the objects: when we think about "A dog is playing with a kid", we are thinking about the dog as a pet because of its friendly behavior. In contrast, in the context of " $A$ dog is sniffing at a drug", we think of the dog as a police dog based on its sensitive olfactory system and dependability. If dogs are taken as inputs in isolation, then their meaning will not differ significantly in the two contexts .

The view that events are processed in their totality can explain why it is so hard to define concepts like the verb, "paint" (Fodor 1981). The premise that events are units of inputs implies that the accurate definition of "paint" will require us to summarize all relevant events in which the action of "painting" is applied for the purpose of covering a surface with paints in specific movements.

Furthermore, studies in developmental cognition have demonstrated that there are evolutionally endowed innate analyzers that can perform parallel object tracing, agent identification, and causal relationship identification, which are the characteristics of episodic events (Carey 2015, 2000). Zacks and Tversky (2001) pointed out that "event representations are (a) structured and (b) closely tied to causes and goals". Therefore, the conceptual system must structurally parse events into agents, patients, actions, and other contextual elements necessary for describing events (Papafragou 2015).

From a teleological view, only an event-based conceptual system can predict the outcome of a given circumstance, which will be impossible if concepts are related to only stand-alone objects. For example, if the concept of a dog is remembered from an event when it barked when meeting a stranger, then we can predict what will happen when a dog encounters a stranger. Although some past research endorses the position that episodic events provide context dependent meaning (Tulving and Thomson 1973), such research does not use episodic events as the basic input units to the conceptual system

Taking episodic events as input units does not exclude the possibility that the conceptual system focuses only on individual objects when it encounters incomplete events. 


\section{Section 1.2 Innate analyzers}

After taking in episodic events, how does the conceptual system further process them? Experimental studies of infants revealed some innate analyzer-based core cognition capabilities in object cognition (Pylyshyn 2001; Pylyshyn and Storm 1988), agency (Saxe, Tzelnic, and Carey 2007; Saxe, Tenenbaum, and Carey 2005), cause representation(Gopnik et al. 2004; Michotte 2017; Schlottmann and Shanks 1992), and analog magnitude innate analysis (Brannon 2002; Brannon, Abbott, and Lutz 2004; Brannon and Terrace 1998; McCrink and Wynn 2007; Xu and Spelke 2000; Xu, Spelke, and Goddard 2005). In Chapter 3 of The Origin of Concepts, Carey (Carey 2000) stated that the innate analyzers have six characteristics: innateness, conceptual content, continuity, learning mechanism, long evolutionary history and iconic format.

A recent research on facial identification further showed that more than 200 neurons encode the structure of a face through the measurements of its parameters such as the distance between the eyes or the width of the face (Chang and Tsao 2017). These neurons output continuous analog values derived through linear algebra-like operations. Given these findings, we can deduce that most innate analyzers output analog values instead of formed concepts. This is very different from the case of the indigo bunting, which learns a representation of north through a special purpose innate analyzer (Emlen 1975). On the other hand, innate analyzers are not perceptual or sensory-motor, they output abstract parameters computed from perceptual or sensory-motor stimulus.

Then a natural question is: "How many innate analyzers are there?" A historical attempt to produce a complete list of them was Kant's table of categories. He identified the categories of quantity, quality, relation and modality (Kant 2002). Modern research has revealed a far more nuanced account of these innate analyzers. There are two distinct systems of core cognition with numerical content. One is the analog magnitude number system, which gives an estimation of the cardinal value of a set (Brannon 2002; Brannon, Abbott, and Lutz 2004; Brannon and Terrace 1998; McCrink and Wynn 2007; Xu and Spelke 2000; Xu, Spelke, and Goddard 2005), and the other is the parallel individuation system (Barner et al. 2008, 2007) which can produce the quantity of small set numbers.

However, there is no guarantee that these works have exhausted all innate analyzers. Wierzbicka (Wierzbicka 2015) argued that the semantic primes found across different cultures can reveal the list of innate input analyzers. After decades of extensive cross linguistic investigations around the globe, researchers came up with a list of vocabularies that are shared across cultures (Goddard and Wierzbicka 1994, 2002; Wierzbicka and Goddard 2014). While the list of semantic primes derived from linguistics has uncovered a lot of categories, it may still miss implicit ones not manifested in human language. For example, most of the $200+$ innate facial feature analyzer outputs are not mapped directly to any linguistic symbols. Increasingly, evidence has shown that many specialized innate analyzers exist (Laurence and Margolis 2015; Tooby and Cosmides 2015). I take the view that the number of innate analyzers must be massive enough to capture all major features of episodic events encountered throughout 
evolutionary history. We can estimate the scope of the innate analyzers through the analysis of neurophysiological data collected from humans and monkeys when they are presented with pictures in many categories. A study has shown that when monkeys and humans are both presented with graphical stimuli of hundreds of objects, a great similarity exists between the dendrograms of the monkey's inferior temporal cortex and the human ventral temporal cortex response patterns (Kriegeskorte et al. 2008). Therefore, innate analyzers must be capable of capturing many important features in the sensory-perceptive inputs. Otherwise, the innate analyzers could not have produced enough distinct response patterns to distinguish all stimulus pictures. Given the small number of semantic primes, we can conclude that a large number of innate analyzer outputs are implicit and not represented by the language system. This also explains why primitive concept meaning is often implicit.

In addition, the consistent response patterns across humans and monkeys suggest strongly that the innate input analyzers are evolutionarily conserved and are highly stable across species. In other words, the system of input analyzers is like a structure consisting of multiple deep neural networks capable of capturing all important features that have been trained and stabilized over the long evolution history.

\section{Section 1.3 Primitive Conceptual nodes}

We can see that innate analyzers output a lot of features, yet many concepts like "color" are related to only a few features. Complex concepts like "dog" are formed based on more characteristics including the 200 plus facial features. However, they still represent only some elements of the whole event. Consequently, we can deduce that there is an abstraction process that reduces the range of focus from all innate analyzer outputs to a subset of them; this process must be the first step in forming a concept. To visualize, we can use one coordinate to represent a single innate analyzer output and the complete set of innate analyzer outputs will form a multidimensional feature space, as shown in Figure 1. Since innate analyzers take whole events as inputs, each event can be represented by a point in the multidimensional space. The projection of each event onto each coordinate will be the feature value of the event in that dimension. The more dimensions we project, the more enriched the event description becomes. The abstraction process can be viewed as a projection from a higher dimensional feature space to a lower dimensional space, as shown in Figure 1.

Innate analyzers like the analog magnitude analyzer (Xu and Spelke 2000; Xu, Spelke, and Goddard 2005) output continuous amplitudes, yet it has been shown that an African gray parrot (Pepperberg and Carey 2012) can learn concepts of one to eight based on their output. This learning process is obviously achieved by dividing the continuous analog output into different segments and then by associating a phonological label to each segment. In the facial feature recognition case (Chang and Tsao 2017), the important finding was that the 200+ neurons encode a set of parameters for differentiating faces. It conclusively denied the long held claim that each neuron encodes one kind of face. Faces of different animals will form different clusters in the space spanned by the $200+$ dimensional features which can be subsequently segmented to form concepts like "dog face" or "cat face". We can induce that 
most innate analyzers are continuous in their outputs, and almost all primitive concepts are formed by segmenting the feature space after the abstraction step.

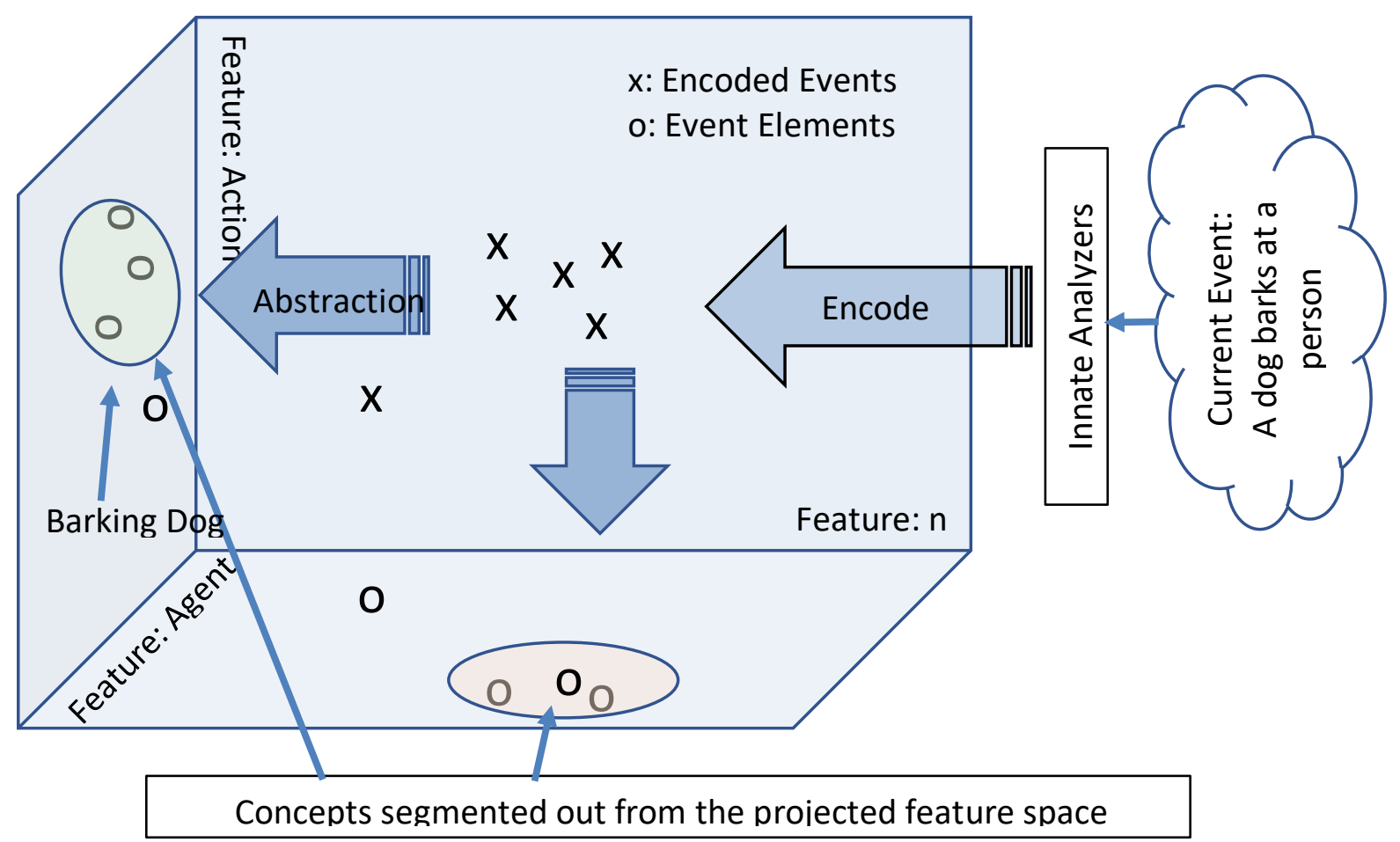

Figure1: Concept is generated by abstraction and segmentation

Because a lot of innate analyzer outputs are implicit and the context of the whole event affects the meaning of their constituent concepts, most concepts derived from innate analyzer outputs are not definable. From this perspective, they are like the primitive concepts (Fodor 1975, 2008). On the other hand, we cannot be sure that all primitive concepts are based on innate analyzers. There is a possibility that some general purpose innate analyzers can be trained to acquire new concepts. However, if these non-innate analyzer concepts are stable in their meaning and are shareable across different subjects, they will behave similarly to innate analyzer concepts. We can approximately equate innate analyzer concepts to primitive concepts.

The important take away from this is that most innate analyzers do not output concepts directly; rather, they create a multidimensional feature space. Natural kinds (Boyd 1999; Brigandt 2009) will form naturally distinctive clusters in the feature space such that when they are discovered, they have naturally defined boundaries for segmentation that varies little from person to person in most cases: we seldom confuse cats and dogs because they are well separated in the feature space of innate analyzer outputs. On the other hand, there are likely to be objects with ambiguous category memberships if they happen to locate on the boundaries between natural kind clusters. 
Given the multitude of features that can be captured by the innate analyzers, a large number of primitive conceptual nodes can be supported. This supports the idea that most lexical items are not definable. The difference between primitive concepts and innate concepts is that primitive concepts are formed from a learning/discovering process while innate concepts are already formed when a child is born. While the former is supported by the proposed the theory, the latter is rejected.

I define a hypothetical entity, a primitive conceptual node, as the basic construct of the conceptual system. The set of features that trigger the formation of conceptual nodes are defined as learning phase features. Based on these features, a set of triggering conditions for activating these conceptual nodes can be obtained by summarizing the range of their segmentation. These learning phase features are grounded to the innate analyzer outputs. Primitive conceptual nodes will be activated if innate analyzer outputs satisfy their triggering conditions. At the same time, since they are also connected to past events, the intersection of

\section{Legends}

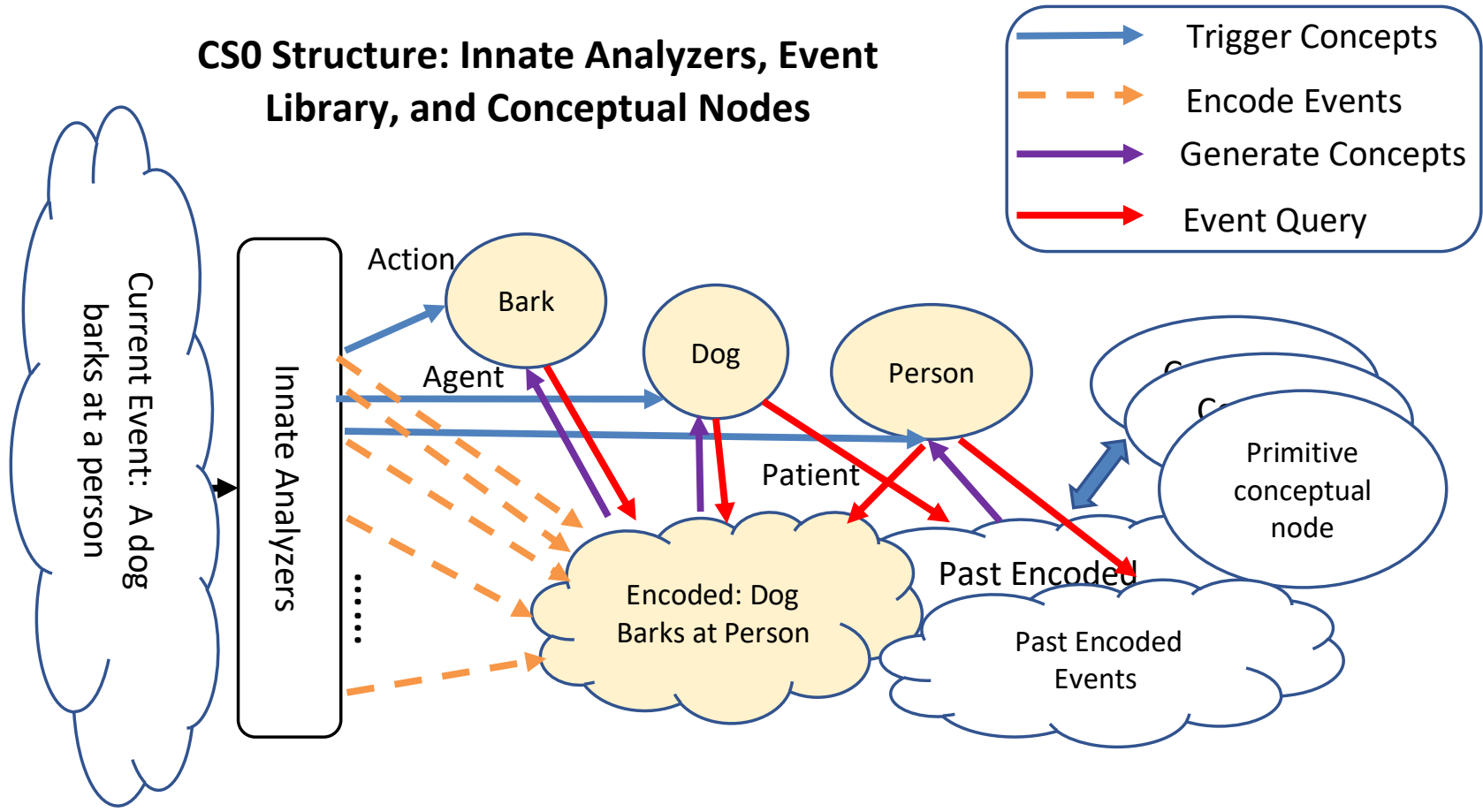

Figure 2: CSO is composed of innate analyzers, past events, and primitive conceptual nodes. Episodic events are encoded as units and primitive conceptual nodes act as indices to them

all events indexed by activated conceptual nodes will be retrieved for event outcome determination. This provides a possible explanation for why sensory-motor neurons will be activated when some concepts are mentioned (Barsalou 2008). At the CSO level, the activation of conceptual nodes activates memories of past events so that associated movements and other information can be retrieved automatically. Primitive conceptual nodes with connections to memorized events can also be viewed as indices to the event library. This process is automatic without any need to involve vocabularies. 
Compared to the stability of the innate analyzers, primitive conceptual nodes are easily shaped-they can grow and change all the time. The proposed system model is different from the deep neuron network models widely used in artificial intelligence, in the sense that conceptual nodes are created from events and act as indices to the events. Consequently, in the proposed theory, information not only flows in the direction from episodic events to conceptual nodes as in deep neuron network models, but also flows from conceptual nodes back to the memorized events Since concepts are triggered by a range of innate analyzer values, it is possible to derive a statistically averaged representation of concepts and their associations, which will form concept prototypes and associative links among conceptual nodes. This lays the foundation for CS1. A concept prototype entails a list of typical features associated with a conceptual node that can be used to identify the concepts and in other functions such as concept composition.

Since primitive conceptual nodes are connected to events, their meaning is naturally contextual because all elements in an event will contribute to the meaning of the conceptual node.

Note that phonological symbols can be associated with primitive conceptual nodes. Consequently, sound stimuli can also trigger conceptual nodes and initiate responses from the cognitive system. Animals certainly use various sounds to communicate, and this has greatly improved their ability to adapt to the environment.

\section{Section 1.4 Event-based reasoning in Conceptual System 0 (CSO)}

Any plausible theory of the conceptual system needs to give an account of what constitutes the basis for reasoning. According to the description in Thinking Fast and Slow (Kahneman 2011), CS1 reasoning retrieves concept associations from the associative memory, which allows fast and automatic responses. Because it rarely involves vocabulary, it is generally considered as pre-linguistic. Since it represents categories by prototypes (Kahneman 2011), it often ignores detailed information. CS1 cannot operate before the establishment of concept prototypes and concept associations. On the other hand, CS2 is generally thought of as the post-linguistic logical reasoning system, which is slower and less error prone. Since it is post-linguistic, it also cannot operate before concepts and associations between concepts are formed. Although we recognize many characteristics of CS1 and CS2, their detailed mechanisms and system structure have not been studied extensively.

There is an important question: how can young babies and adults reason when they encounter novel situations for which few or no concepts are available? Very young infants have been observed to accomplish very sophisticated false-belief tasks (Wellman, Cross, and Watson 2001; Wimmer and Perner 1983), yet what forms the innate support for logic tasks is virtually unstudied (Carey 2015).

I argue that there must exist a pre-linguistic and event-based reasoning system, termed as conceptual system 0 (CSO) prior to the formation of the concept-mediated reasoning systems, CS1 and CS2. I hypothesize that past events parsed by the evolutionarily conserved innate analyzers are stored, organized and indexed by primitive conceptual nodes so that they can be 
used for predicting event outcomes in CSO. For example, upon seeing a dog and a stranger, CSO will retrieve the events indexed by both "dog" and "stranger" to predict that the dog will bark. Alternatively, if we see that a dog is barking, we can reason that it could be caused by the presence of a stranger. In other words, reasoning is mediated through memorized events in the proposed CSO theory, which can explain some very sophisticated cognitive behaviors in animal and human studies.

\section{Event Mediated Reasoning in CSO}

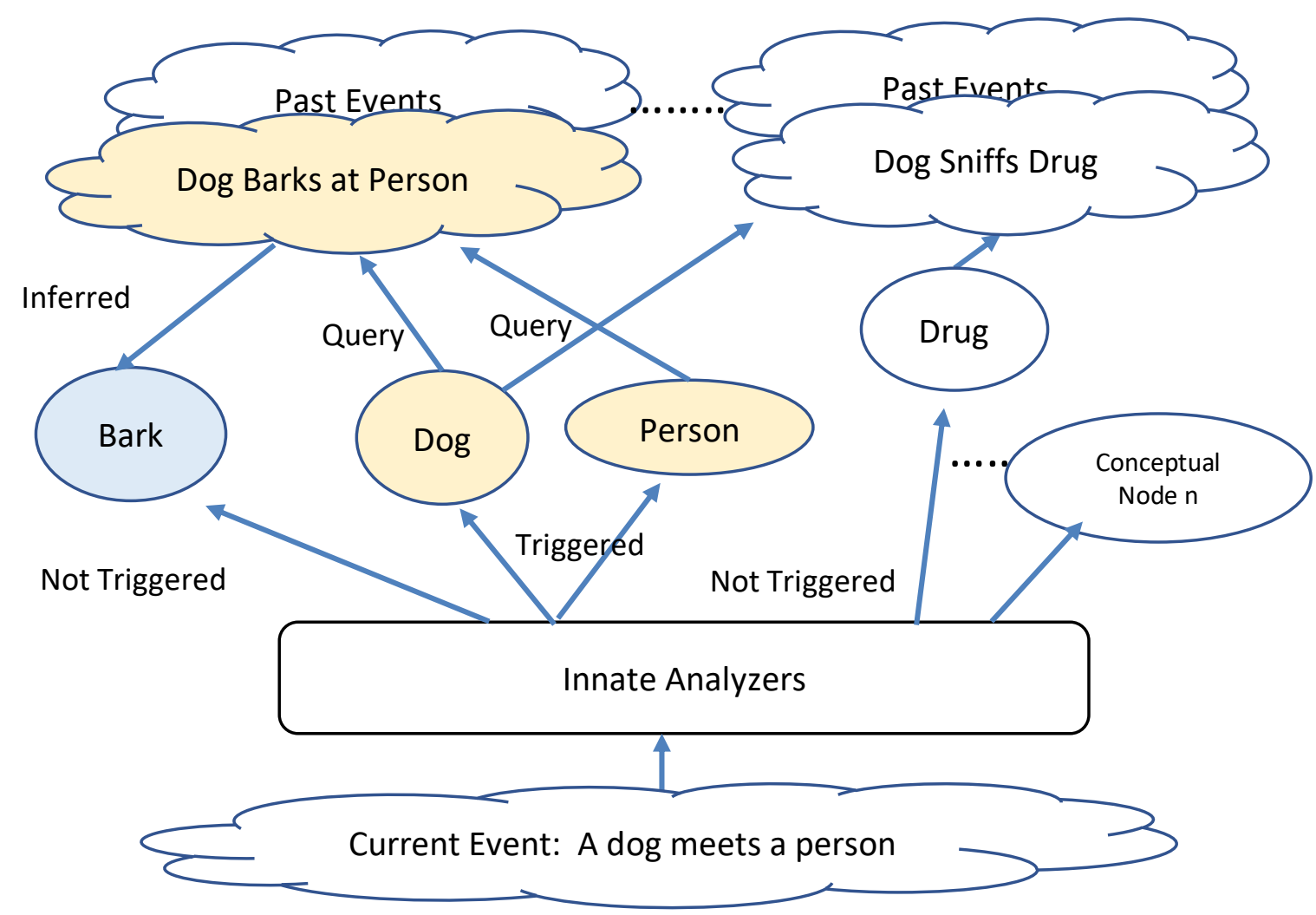

Figure 3: Event Mediated Reasoning in CSO example. Given the current event that a dog meets a person, the conceptual nodes "dog" and "person" are activated. Then the past event that involves the activated conceptual nodes "Dog barks at a person" is retrieved. Based on this event, CSO predicts that the dog may bark next.

Studies (Tse et al. 2007) showed that rats can learn flavor-location associations more quickly after an initial training period. At the beginning of each training session, rats in one of the four starter boxes situated on the outside periphery of an arena were given small pieces of food in a certain flavor. Then the rats were let out to explore the arena. Larger quantities of food in the same flavor were placed at one of the six sand wells. The training was repeated several times for each flavor until the rats had learned all six flavor-location associations. In a later testing session, if the rats were given a cue for any one of the flavors in a random starter box, they would go to the associated location with a significantly higher probability than by chance. For each association, the learning process usually required 10-12 training sessions. However, after 
the initial training, when two new flavors associated with two old places were introduced, each of the new associations was learned in a single training session.

In the complementary learning systems theory (CLS), the standard interpretation of such findings is that animals possess a "rapid learning mechanism" in the hippocampus region and a "slower learning mechanism" in the neocortex region (Kumaran, Hassabis, and McClelland 2016). To further prove this hypothesis, hippocampal lesions were introduced in a group of rats 24 hours after learning the new flavor-location associations. Remarkably, this group still retained all associations including those rapidly acquired ones. However, the group lost the ability to learn new associations no matter if the flavors were associated with old locations or new locations. In contrast, the control-group with sham lesions could still learn all kinds of new associations although the speed of learning did not improve despite previous training. A later study suggested that there are separate rapid and slow learning processes (Tse et al. 2011) and the existence of an old "schema" of flavor-location association can assist the rapid learning process. However, this explanation is unsatisfactory for several reasons: In what way does the old "schema" assist the learning of new associations? Why is the assistance obvious when old locations are involved yet there is no difference when new locations are involved?

The observations in the flavor-location learning experiment can be explained by the proposed CSO model and its operational principles: rats were first exposed to multiple events of "Finding food of flavor $F$ at location $X "$ during the initial training sessions. Based on these events, they formed conceptual nodes of all locations and all food flavors. Each flavor and each location were first associated based on the events in which they co-occurred. The slow speed in the initial establishment of these flavor-location associations can be attributed to the difficulty in generating the locational conceptual nodes. At the same time, the repeated training sessions also allowed the establishment of direct flavor-location conceptual node links in the associative memory through a statistical averaging process at the CS1 level. On the other hand, the establishment of new flavor conceptual nodes must be very fast because rats could learn new flavor-old location associations within a single training session.

The rapid learning and application of the new flavor-old location associations proves: 1 . Old location info must have been extracted and stored in a conceptual node during the initial training period. Otherwise, it would be impossible for the rats to directly navigate to old locations when they are cued by new flavors after only one association training session 2. New flavor-old location associations could not have been established in the associative memory, which requires statistical averaging over several training sessions. 3 . The rapid learning of new flavor-old location associations must have been enabled through event-based reasoning; after a single training session, the conceptual node of the newly introduced flavor could act as an index and all relevant past events were retrieved when the rats were cued. Although there was only one such event in their past experience, it nonetheless contained the relevant event for activating the conceptual node of the old location. Subsequently, the "schema" of navigating to this location was retrieved so that the rats could find the food with the new flavor. 
In my proposed theory, if the mechanism for establishing the links between conceptual nodes and episodic events is destroyed, then new conceptual nodes can no longer be generated based on new events and old conceptual nodes can no longer be linked to new events. This provides a probable explanation for why hippocampal lesions disabled both rapid and slow learning of new associations. This account corroborates well with the most widely supported hippocampal indexing theory, which states that the hippocampus forms an index of the cortical activity that was present during the actual experiencing of an event and that the contents of component memories are actually stored in the distributed cortical networks in which this activity took place (Damasio 1989; Teyler and DiScenna 1986). Past studies have also shown that hippocampal-lesions can cause impairment to between-domain associations (Mayes, Montaldi, and Migo 2007) in human patients with relatively selective hippocampal-lesions (Holdstock et al. 2002). The proposed theory gives a satisfactory account for all these findings. The actual mechanisms for the forming and retrieval of episodic event memories are still the subject of active research. Multiple regions including hippocampus and medial prefrontal cortex are found to be associated with new episodic event memories and the possibility that both are involved in remote episodic event memory retrieval cannot be ruled out (Tonegawa, Morrissey, and Kitamura 2018).

The finding on how new flavor-old location associations can be learned rapidly and retained, even after the introduction of a hippocampal lesion shortly after learning, posed a big challenge to the CLS theory, which assumes that newly acquired memories will remain hippocampusdependent for an extended time to allow for gradual interleaved learning in the neocortex. Although this difficulty can be overcome if we assume that the neocortex is like a deep neural network that is capable of fast learning of new information consistent with old knowledge, while the hippocampus only replays new episodic events to the neocortex (Kumaran, Hassabis, and McClelland 2016; McClelland, McNaughton, and Lampinen 2020). However, such a model is fundamentally empiricist, which contradicts the increasing evidence that supports concept nativism. Also, it cannot offer an account for the phenomenon of discontinuity in concept learning (Carey 2000).

Despite the various interpretations of hippocampal-lesion experiments, they all agree on the existence of two prelingual conceptual systems. These cannot be treated as the equivalent of the fast and slow human reasoning systems because both are prelinguistic and do not support rule-based logical reasoning. Consequently, this provides strong support for the proposed claim that the human conceptual system is three-tiered with two prelinguistic systems and one postlinguistic system.

Another argument for the existence of CSO is that it can explain why there are inexplicable contextual meanings of words that can be sensed yet not explained. An example of this phenomenon is the device of imagery in poetry, which uses words to evoke vivid mental pictures. In such cases, words do not even have to be organized into complete and coherent sentences. As long as they can evoke mental pictures of past events, then they can be effective. Imageries cannot be explained by CS1 which tends to omit explicit or implicit contextual information: "System 1 is radically insensitive to both the quality and the quantity of the 
information that gives rise to impressions and intuitions" (Kahneman 2011, p. 86). This is not surprising given that CS1 statistically averages both the content of conceptual nodes and the links among them. Although the knowledge system in CS2 theoretically can accommodate explicit contextual information of events, it cannot explain inexplicable contextual meanings of words because it relies on language to construct descriptions of events and contextual information and always requires clear and coherent representations.

Only in the proposed CSO theory, the ontology of concepts are mental representations of encoded events, which can be triangulated and retrieved by multiple activated concepts. Therefore, even scattered words in poetry can still evoke vivid mental pictures that can be effective in conveying meanings.

The proposed CSO theory is also compatible with embodied theories of concepts because conceptual nodes are connected to the memory of events. Consequently, the activation of concepts will also allow us to experience past events, which has been demonstrated by many experiments (Barsalou 2008).

In summary, CSO is defined as the system composed of primitive conceptual nodes that act like indices to memorized events. This system is evolutionarily conserved in the sense that it is constructed from innate analyzers shared across species. This system is capable of basic "reasoning", i.e. the prediction of event outcomes so that proper reactions can be determined in various situations.

This system consists of a lifelong and stable layer of innate input analyzers that are largely determined by genetics, while the rest of the primitive conceptual nodes are flexible and are generated through exposures to experiences.

\section{Section 2: CS1 structure and reasoning}

CS1 reasoning has been characterized extensively in the literature. In this paper, I focus on elucidating its structural composition so that its behaviors can be explained. CS1 is characterized by a fast reasoning process based on activated concepts, their common associations and emotional states (Tulving, Schacter, and Stark 1982; Caruso, Shapira, and Landy 2017; Berger, Meredith, and Wheeler 2008; Vohs, Mead, and Goode 2006; Bolte, Goschke, and Kuhl 2003). It tends to ignore nuances in information and is biased to familiar concepts with cognitive ease (Oppenheimer 2006; Kahneman 2011). Repetition increases liking and reduces cognitive difficulty (Zajonc 2001). It is error prone and adheres to norms (Oppenheimer 2006; Kahneman 2011; Kahneman and Miller 1986). It is largely an automatic and unconscious process.

CS1 tends to see patterns and causal relationships (Michotte 2017; Hsu 2008) to make up coherent stories without deeper reflection (Mednick 1962; Hsu 2008). For example, according to Chapter 10 of Thinking, Fast and Slow (Kahneman 2011), if people hear that some rural counties have the highest pancreatic cancer rate, they might make up a story that it must be a 
higher alcohol consumption rate that leads to the high pancreatic cancer rate. However, it is also true that the lowest pancreatic cancer rates also happen in rural counties and the phenomenon is actually due to larger statistical variations that exist in counties with a smaller population. This capability of making up hypotheses cannot be attributed to CSO event-based reasoning; therefore, I argue that there must exist a Dynamic Event Composition Module (DECM). Given that CS1 tends to ignore hidden information and has a characteristic called What You See Is All There Is (WYSIATI), this DECM module must take only currently activated conceptual nodes as its inputs. Also, since CS1 relies on associative links between concepts in reasoning, DECM must also use conceptual nodes activated through associative links as inputs. Given that CS1 ignores nuances and makes judgements based on norms, the DECM must take concept prototypes as its inputs. Since the emotional state affects CS1, DECM must take emotional states as its inputs. Given that the emotional state and activated concepts are time changing, DECM must be dynamic in its responses. In composing events, DECM must actively seek to assign roles such as agent, patient and movements to active conceptual nodes.

Since statistical averaging requires several examples, CS1 must be constructed based on CSO by 1. Generating concept prototypes through statistically averaging relevant concept features, 2. Adding links among conceptual nodes in the associative memories based on their frequency of co-occurrence in the initial training period, 3. Generating fast event outcome predictions based on DECM.

It is speculated that during the evolutionary process, animals developed CS1 reasoning to handle sudden dangers (Kahneman 2011). Animals cannot rely on event-based reasoning, which requires the retrieval of all past relevant events. Instead, they will quickly activate associated concepts and construct a story based on them. For example, upon hearing a barking sound, the conceptual node "bark" and it frequently associated node "dog" will be activated. These two nodes will in turn lead DECM to quickly compose the likely event that " $A$ dog is barking".

CS1 is automatic and unconscious because the conceptual nodes are triggered by innate analyzer outputs or associative links. Emotional states can influence the outcome of DECM just like other conceptual nodes.

DECM is the module that allows imagination because it can create non-existent events based on activated concepts. In the past, some researchers equated creativity with the ability to make coherent stories from remotely associated concepts (Mednick 1962).

\section{Section 3.1 CS2 structure and Conceptual nodes in CS2}




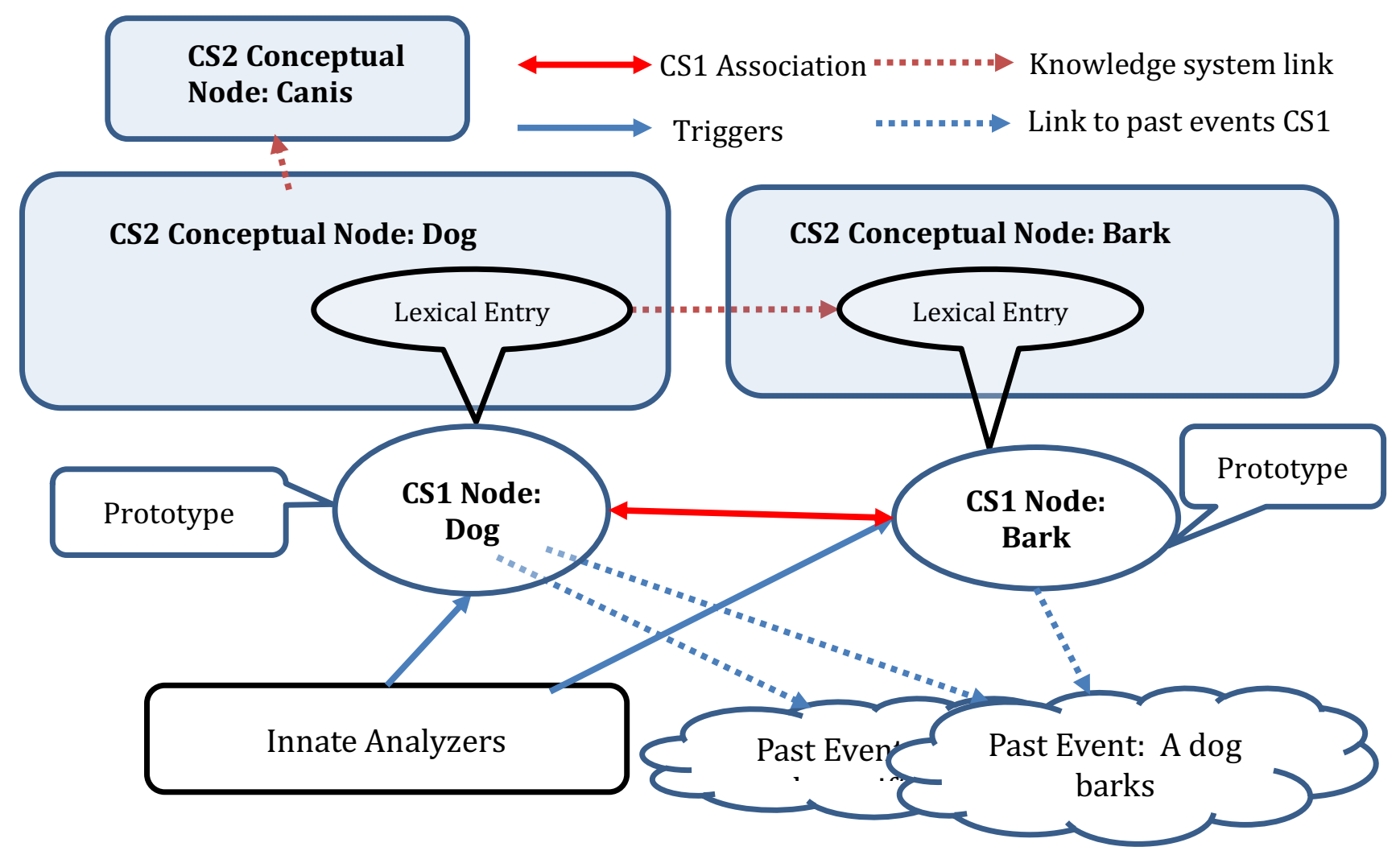

Figure 4 The conceptual node structure in CS1 and CS2. Each CS1 primitive conceptual node is annotated by it prototype. Primitive conceptual nodes can be linked to their CS2 conceptual nodes through a Quinian bootstrapping process. Any CS1 conceptual nodes can be activated by innate analyzer outputs, its lexical representations, and its associative links.

In CSO, conceptual nodes are viewed as indices to events. In CS1, conceptual nodes are further annotated by prototypes and associative links. In CS2, primitive conceptual nodes established in CSO can be further linked to morphological and phonological symbols. All these associations provide different ways to trigger the primitive conceptual nodes as well as innate analyzer outputs. Primitive conceptual nodes can be discovered when they are aligned with linguistic symbols in a Quinian bootstrapping process(Quine 1960, 1969, 1974).

In addition to "discovering", CS2 can create conceptual nodes and their associations through learning directly. In fact, many scientific concepts are first taught as a set of related morphological and phonological symbols unrelated to past experiences. For example, in physics, we learn that atoms consist of electrons and protons even though we have no tangible experience with them. This shows that the cost of creating and establishing new mutuallylinked linguistic CS2 conceptual nodes is rather low.

The key difference between CS2 and CSO/CS1 is that new linguistic conceptual nodes annotated by phonological and morphological symbols can be linked to past events and other conceptual nodes with great flexibility that enables the creation of various data types. First of all, similar conceptual nodes that vary in some feature dimensions can be grouped together to create 
super nodes, which makes it possible to create category/kind concepts. For example, by grouping all number concepts together, the category/kind concept of quantity is formed. Not only does such a concept organize the conceptual system, it also allows the creation of a variable conceptual node whose value can be set by any concept under its category. For example, the category of integers allows the generation of an integer variable whose value can be set to any of its member integer values. Variable concepts are possible only in CS2. For example, the concept of eye distance is abstracted from the category of animals with two eyes. It is not a primitive concept because it is not generated from innate analyzer outputs. It is generated from the kind concept of animals with two eyes. Yet the variable of eye distance is different from the kind concept of animals with two eyes in the sense that it is focused on only one feature of the kind concept.

The flexibility of CS2 also allows creation of temporary connections between nodes and allows conceptual nodes to function as shifting pointers to other conceptual nodes and encoded events. For example, any integer value can be labeled as the "current number" in a counting task. The concept of today, yesterday, and tomorrow are examples of pointers that shift their referents every day. The flexibility in creating links between conceptual nodes also allows the creation of linked lists so that we can store lists of integers of one, two, three... or Monday, Tuesday, Wednesday... in our memory. Relational concepts such as "have" can also be used to clarify the relationships among concepts. Given relational concepts, it also becomes possible to store knowledge systems such as those for a car, as discussed by Evans (Evans 2015).

The flexibility in constructing conceptual nodes and establishing links between nodes and events in memory allows CS2 to create most data types that you can find in a modern computer system, which are the basis for complex logical thinking.

\section{Section 3.2: Post-linguistic CS2 reasoning-logical reasoning}

Logical reasoning (van Dalen 2004; Meyer and van der Hoek 2004; Kant 2002) is obviously a huge topic for which I cannot give a thorough account in this paper. My intention is to generalize logical reasoning at a level such that we can identify the set of components and functional modules. In this way, the implication of the proposed conceptual theory on logical reasoning can be elucidated. Generally speaking, logical reasoning can assume the form of deductive, inductive, abductive, analogical and statistical reasoning for elucidating either the antecedent, contextual, or concluding conditions of concerned events.

The functions of CS2 are organized around the need to generate hypotheses of laws/rules and their applications. Logical reasoning requires the conceptual system to have at least the following functionalities: 1 . Recognize and generate categories of concepts 2 . Generate hypotheses of laws that characterize how certain feature values covariate with other nonobvious factors. 3. Compare and validate category membership of concepts to see if a law/rule can be applied. 4. Compare composed events by DECM with known laws to judge their validity. I argue that there must exist a Rule Forming and Checking Module (RFCM) in CS2. 


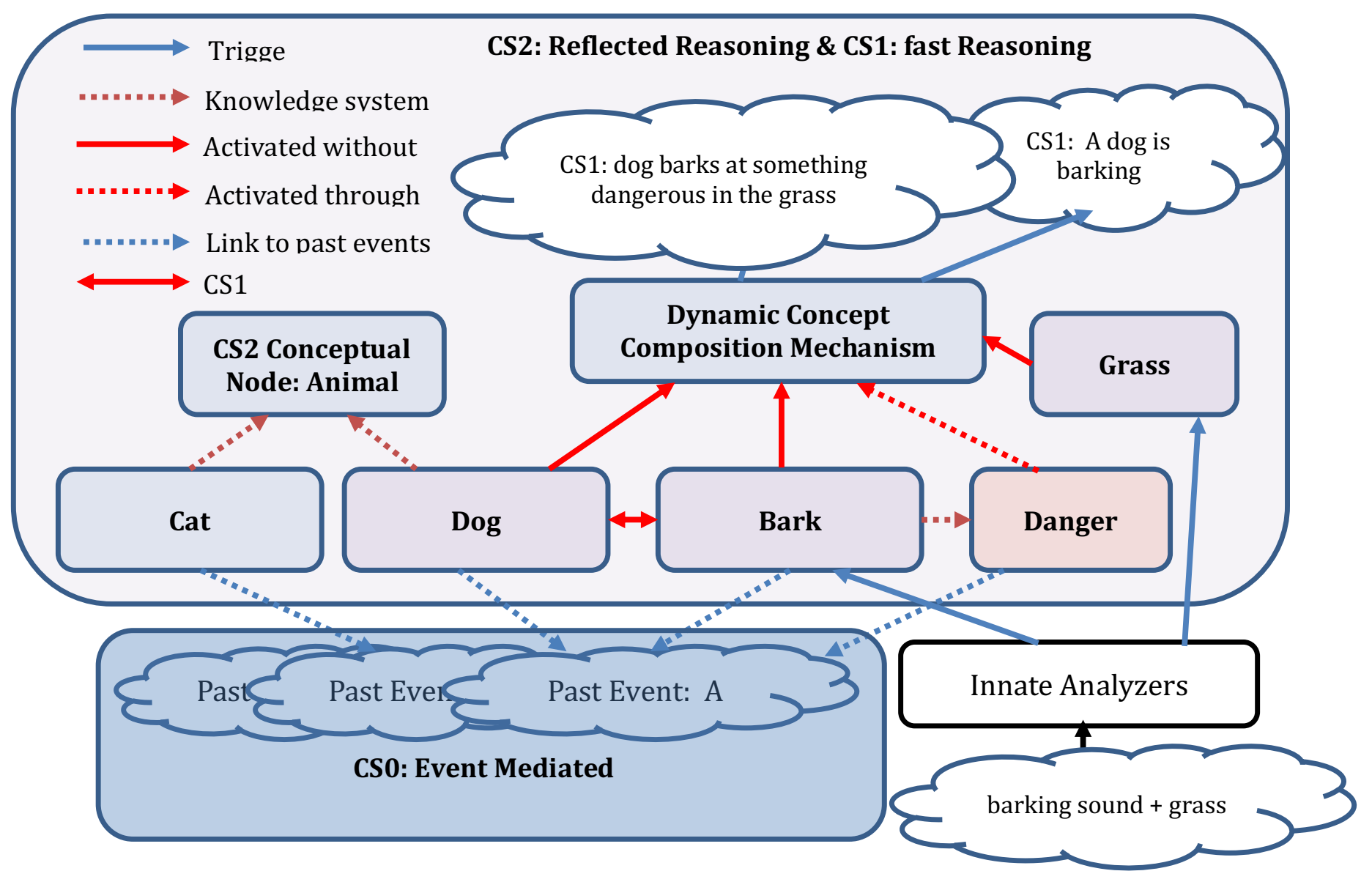

Figure 5. CS1 and CS2 Reasoning. CS1 reasoning quickly activates relevant conceptual nodes and compose a likely event as the basis for reasoning. CS2 further employs the rules stored in the knowledge system and accepts or rejects the composed hypothesis by DECM

Given the sketched functionalities, we can further delineate the characteristics of RFCM. Since this is also a rather big and difficult topic, we can start from an example and examine the set of operations needed to accomplish it. Consider the ability of generating hypotheses of laws that characterize how feature values vary with other non-obvious factors. Without employing metaphorical reasoning, this task needs to $\mathrm{f1}$ : gather a list of relevant events that contain the feature value of inquiry; $f 2$ : extract values of the concerned feature and other possible correlated features; f3: estimate feature correlation through computation; f4: Propose a hypothesized law of how one feature changes with another feature through induction and test it on new relevant events.

We can see that this RFCM is uniquely CS2 because none of such functions are present in CSO or CS1 and it requires a working memory and variables for these manipulations.

Besides rule forming, another important aspect in CS2 is the ability to compare composed hypotheses with known rules and laws. For example, we see a barking dog and only grass is in front of it. We will check the hypothesis "a dog is barking at the grass" with the rule that "a dog 
will bark at another person or animal". This results in a violation of the law. Subsequently, all of these activated nodes are picked up by DECM and it composes a new hypothesis that "the dog is barking at an invisible small animal in the grass" that conforms to the law. So CS2 reasoning can be viewed as the process of using deeper reflections based on the knowledge system and composing events that satisfy all known laws.

In this paper, I have made an initial attempt to delineate the components and functional modules required for CS2 logical reasoning. The discussion of many other functions can be left for future research, which will require a significant undertaking.

Since logical reasoning is conducted using concepts triggered by one or several feature values, their limited power to represent the underlying event shall be considered carefully. Only when features not involved in the logical reasoning process are stable and non-consequential to the status of the relevant events, then logical reasoning can lead to reliable conclusions. Otherwise, they may lead to fallacy.

For example, integer addition is developed to account for the changes in quantity when individual items are added together while other physical or chemical properties of the items are stable and non-consequential. In such cases, integer addition is enough to account for the change. If other physical or chemical properties are not stable and adding items together incurs the merging of the items, then integer math is not enough to describe the changes.

Besides the issue of limited features of concepts, logical reasoning often runs into issues when concepts are defined at the extremes of the feature space. For example, if we examine Kant's antinomies (Kant 2002), the famous breakdowns of logical reasoning, we can see how such a phenomenon is formed using the proposed concept-forming theory. His first antinomy is about whether the world has a beginning in time, for which he found logical reasoning for both the thesis and the antithesis. For the thesis, he argued that the world must have a beginning in time because otherwise, the world has to finish synthesizing an infinite series of successive states of things to reach a point in time, but the infinite series cannot be completed through successive synthesis, consequently, there must be a beginning in time.

For the antithesis, he argued that if the world has a beginning in time, then the world has to be born out of the empty time; however, no part in this empty time has the distinguishing condition for existence. Hence, the world cannot be born out of this empty time, so the world does not have a beginning in time.

The above proof implicitly assumes that the rule governing space and time of this world will still hold in the extreme case of infinity, which is not a given and has to be proved before we proceed with the rest. Even if we ignore these factors, the proof still cannot stand.

As we have stated, concepts are formed when the underlying feature space is segmented. So, our concept of time infinity is formed by segmenting the continuous time dimension at an imagined limit. This segmentation will result in two concepts which easily can be confused with 
one another. Beyond the limit, the infinity of time cannot be reached, and below the limit, the infinity of time can be reached by traversing through the successive synthesis of things.

Now if we analyze Kant's proof for the thesis that the world has a beginning in time, we can see that it employs the double negation law. It essentially tries to prove proposition $\mathrm{P}$ by arguing "not (not P)," in which P is "The world begins at a time point that can be reached backward through successive synthesis of things". "The world has no beginning in time" is thought to be the opposite of P. However, this treatment is problematic. "The world has no beginning in time" means either that the world has a beginning but the beginning of the world cannot be reached or that the beginning of the world is not definable in the feature space of time. If it is the latter case, then the ensuing arguments about how it is not possible to traverse through eternity to the current time is invalid because it is equivalent to taking the null event "the world has no beginning in time" as the opposite to "the world has a beginning in time". To make an analogy, it is like saying that a colorless object cannot have a color value, because if it has a color, it must have a color value, and therefore, it cannot have a color value. This is of course a circular argument.

If Kant's proof meant to say that the world began at a time that is in the infinite past which cannot be reached and it is the opposite of the concept of a time point that is reachable backward, then the problem is that the boundary dividing the two concepts is an imagined one which can be arbitrarily moved up or down. Consequently, the two concepts are not true opposites and the proof of the thesis is invalid.

The problem in these arguments arises when we segment the feature space to generate the concept of infinity, yet the segmentation boundary is not clearly defined. This explanation supplemented further details to the original set of critiques of reason that attributed the phenomenon of antinomies to applying reasoning beyond experiences.

\section{Section 4. Concept Composition}

Among higher level cognitive functions, forming complex concepts from primitive ones is of critical importance. A valid conceptual system theory has to give an account of how concepts are composed that can reconcile various phenomena observed in concept composition. The main issues associated with concept composition arise from two aspects: 1 . Without a unified theory on what is contained in concepts, it is hard to know what "ingredients" are used in concept composition; 2 . Without an agreement on what impinges on the meaning of composed concepts, the process of concept composition that constructs meaning is also unclear.

Regarding the first problem, it has been shown that prototypes containing typical features are retrieved and used for concept composition (Hampton 1987, 1997; Huttenlocher and Hedges 1994). Furthermore, modal and causal knowledge (theories) affect concept composition (Hampton 1997; Murphy 1988, 1990; Keil and Wilson 2000). This picture is further complicated when it is noticed that some features not represented by the prototypes of component concepts will emerge in the combined concept (Kunda, Miller, and Claire 1990; Fodor and 
Lepore 1996). Some attributed this to exemplars (Medin and Shoben 1988; Hampton 1987) that refer to the same phenomenon of extensional feedback (Barsalou 2017) or the guppy problem (Osherson and Smith 1981). In addition, multimodal simulation (Wu and Barsalou 2009) and distributed linguistic representations (Barsalou et al. 2008; Louwerse and Connell 2011; Glaser 1992; Baroni and Lenci 2010; Erk 2012; Landauer and Dumais 1997) are all found to be contributing factors to concept composition. Consequently, the discussion of what concept content is involved in composition has to be nuanced and organized under various types of composing processes.

Regarding the second problem, ontological compositionality (Pelletier 2017; Boodin 1939) states that the meaning of the whole is contained in its parts (Fodor and LePore 2002). Opposing this view, functional compositionality takes the position that the meaning of the whole can be different from what is in the part. Some argued that component concepts and the way they are arranged act like arguments for a function that outputs sentence meaning (Pagin 2012 , 2009). Besides the character of the concept composition process, research further reveals that it does not follow the rules of set logic in combining features of individual concepts. Instead, it is the interactive combination of intentional meanings of individual concepts that constructs complex concepts (Hampton 2017, 1988). Some detailed properties of concept composition have been elucidated: concept composition is not commutative and one of the concepts carries more weight and dominates. Research on how people combine concepts showed that 1 . They instantiate superordinate categories to basic level categories; 2 . They align properties and functions between the two concepts; and 3. A simulation process can be involved if there has never been an example of the concept to be composed (Barsalou 1991).

Despite this progress, many questions remain and the research has been limited mostly to the composition of two words. Pelletier summarized the list of problems, among which the most interesting one is "how can mere words combine to form sentences", which is noted as a different problem from noun-noun, adjective-noun combination problems. While a noun-noun or adjective-noun combination results in other concepts, a sentential combination will not result in other concepts because that would require a further explanation of how the resulting concepts can form a sentence. This puzzle is called the Bradley's Regress puzzle and it has not been adequately addressed (Pelletier 2017).

I argue that much of the confusion arises from a lack of understanding of "the meaning of the meaning of composed concept", which makes it hard to understand what concept content goes into the process of concept composition and what is the goal of concept composition. In this paper, I propose that the meaning of a composed concept or sentence lies in its set of active features and how these active features are engaged in mutual interactions. This interactivefeature theory for concept meaning is based on 1 . The conceptual system takes episodic events as input units for processing and a sentence/phrase completely/partially represents an event. 2. People try to fit the features of individual concepts together when composing concepts (Hampton 2017), which shows that the goal of concept composition is to find a list of features that interact with one another in a coherent way. 
Indeed, we judge the understandability of a sentence or phrase by the degree of how much we can understand the interactions intended by the composed sentence or concept. To understand a composite sentence such as "He held onto a basketball in water" is to understand that the basketball can float and this feature interacts with the man who could otherwise sink into the water. We claim that we understand this rather uncommon sentence because we can envision how some features of the basketball can interact with the man. On the other hand, "A goldfish held onto a basketball in water" will not make sense because a small goldfish does not have any feature that allows it to interact with a basketball in water by holding it. The understandability of a composed sentence or phrase depends on if there is a conceivable way such that the involved concept features can interact with one another.

This view is quite different from other accounts of concept meaning that are mostly discussed in a rather sketchy manner. For example, some scholars think meaning lies in our mental representations of the world (Fodor 1975, 1983, 2001), while others argue that meaning lies outside of our heads (Putnam 1975; Kripkie 1980). Proof-theory semantics believes that meaning is a kind of proof derived formally through deductive logic (Francez 2015); Semantic holism takes the position that a concept's meaning lies in its place in the entire network of liaisons among the concepts (Pelletier 2017). A method for examining these theories is to check how well such theories can explain the fact that concept meaning always changes with different contexts (Evans 2009). In this aspect, the proposed interactive-feature theory holds up well: since the set of engaged features of a concept in different contexts always changes, its meaning will always change in different contexts.

Since all activated features of involved concepts matter, three sources can contribute to the meaning of a composite concept. The first source is the set of mutually engaged features of all concepts in the context. Since features of other concepts will determine how they interact with the features of the current concept, all activated concepts in the context will influence the meaning of a specific concept. The second source is the activation/identification features of component concepts that will vary in each instance. For example, we can invoke the concept of a rabbit by its front look, its movements or its name. The third source is the set of features associated with its constituent concepts when they are first learned or created. Features acquired during the learning process are always invoked regardless if they are engaged with other concepts in the context or not (Hoenig et al. 2008) .

On top of clarifying the "ingredients" that go into concept composition, I argue that the process of concept composition is the process of constructing how the involved features will interact with one another. This composing process can also be viewed as our hypothesis generation process that initiates the reasoning process. Since CS0, CS1, and CS2 are all involved, concept composition should be discussed separately in the three tiers. Otherwise, conflated discussions will lead to contradictory accounts.

Within the proposed system structure at the CSO level, concept composition easily can be explained as the process of finding the intersection of events indexed by all component concepts. For example, given the word "pet" and the word "bird", we will look for the set of 
events that are linked to pets (animals raised by humans) and birds (animals that have wings). Then an example event with a pet bird may emerge. Features important to keeping a bird as a pet such as a cage become part of the meaning of the composed concept. Note that this account is relatable to past theories of concept composition based on exemplars (Medin and Shoben 1988; Hampton 1987). However the difference is that exemplars are considered as entities outside of their specific contexts; while in event-based composition, concepts are indices to events and consequently also activate context information embedded in the events. As to extensional feedback (Barsalou 2017) and case-based reasoning (CBR) (Hammond 1990, 2012), they are somewhat similar to the proposed theory, except that the proposed theory explicitly designates conceptual nodes as the indices to past events that have an inherent structure consisting of agents and patients, etc. In contrast, CBR has been developed mainly in the field of artificial intelligence. Numerous indexing methods have been proposed. Earlier proposals of CBR index the cases by their goals and how their features differentiate them from other cases (Hammond 1990). Later, some researchers built indexing to cases through domain specific ontology (Gao and Chen 2012).

This account of CSO concept composition does not require the merging of prototypes, which has been one of the main difficulties facing prototype-based theories because they cannot explain the problem of new emerging features such as "cage" in the composite concept of "pet bird" (Fodor and Lepore 1996; Grandy 1990). In the past, such a difficulty has posed great challenges to the prototype theory.

In CS1, concept composition is done through the activation of associated concepts that are taken up by DECM. Typical association between concepts implies a set of typically engaged features that interact with each other, and the activation of DECM allows the transformation of concepts into an simulated event that can be expressed as a sentence in the linguistic system. This account solves the Bradley's Regress puzzle.

In the past, cognitive psychologists have framed the problem of distributed linguistic representation as utilizing existing associations between words for concept composition. This has been applied in tasks such as "modeling word similarity, judgments, discovering synonyms, concept categorization, predicting selectional preferences of verbs, solving analogy problems, classifying relations between word pairs, harvesting qualia structures with patterns or example pairs, predicting the typical properties of concepts, and classifying verbs into alternation classes" (Baroni and Lenci 2010). However, such research has not been directed towards finding the specific concept features engaged in composed concepts as a way to understand them.

Since CS2 reasoning involves logical thinking, concept composition can be conducted as logical combinations of the list of features of constituent concepts that are clearly defined, such as those in math.

An interesting problem is the composition of counterfactual concepts that cannot find any past events linked to their constituent concepts. For example, "unmarried worm" is a counterfactual concept for which we have zero experience and it cannot be composed in CSO. 
Also, CS1 associative links between such unusual combinations of concepts do not exist, so the process of such compositions can only be initiated in CS2.

If the feature dimensions of the constituting concepts overlap, then we can compose an imagined concept. For example, we have no problem imagining a man with blue skin although we have never met one. This is because in the concept of man, its defining feature space includes the dimension of skin color, which can be replaced by blue. In contrast, the thought of "unmarried worm" comes up with an empty mental representation because "worm" is not defined by the dimension of marriage. This combination process may be conducted by definitional combination or through DECM. It is highly likely that both CS1 DECM and CS2 are involved in the process.

Also, since conceptual nodes associated with events have prototypical representations, it makes the imagination of events based on these prototypes possible. Suppose we have the prototype of a human, barking, and a dog. We now can imagine the event of a man barking at a dog although we have never experienced it. This example reflects the view that prototypes consist of a set of feature-value pairs (Hampton 1979, 1988) Feature value replacement has been discussed as a selective modification model (Keane 1999) based on prototypes, which suggests that such compositions are conducted at the CS1 level.

The proposed three-tiered theory points out many possible methods for concept compositions. Future research on concept composition will have to distinguish the characteristics of different concept composition mechanisms so that we will no longer deny prototypical composition in CS1, based the evidence of new emerging features from CSO concept composition (Fodor and Lepore 1996).

\section{Section 5: The relationship between CS0, CS1, and CS2 systems}

CS1 and CS2 reasoning can be conducted simultaneously in parallel (Evans 2003). I further extend this conclusion to include CSO. For example, conceptual nodes triggered by perceptual stimuli may lead to the further activation of other associated conceptual nodes. These activated nodes can be used to retrieve relevant events in event-based reasoning in CS0 or through CS1 DECM. In case there is a conflict, CS1 is likely to be prioritized. If the CS1 prediction is challenged, we may further reflect using CS2 logical reasoning.

The coexistence of CSO, CS1 and CS2 can explain some of the discontinuities in conceptual development observed in past studies (Wellman, Cross, and Watson 2001; De Villiers 2005). For example, a false-belief experiment (Wimmer and Perner 1983) was set up such that during habituation, babies could observe that some food was hidden at place $A$ in front of the eyes of an actor. Then the actor left the scene and the food was moved to a new hiding place B. It was believed that if the babies have theory-of-mind capabilities, they can correctly predict that the actor would look at place $A$ and would be surprised if the actor looked at place B when the actor returned. Indeed, many studies have confirmed that preverbal babies do have this ability (Onishi and Baillargeon 2005; Surian, Caldi, and Sperber 2007). However, at an age between 2 
to 4 , young children fail this test. It is only after age 4 that they can regain the ability. The controversy surrounding this experiment is whether preverbal babies really do have theory-ofmind capabilities in representing the mind state of the actor or does some other simple explanation exist.

It is entirely possible that preverbal babies can successfully carry out false-belief tasks based on the proposed CSO theory. During habituation, the event of "An actor saw food being hidden at place A" allowed the babies to develop the concepts of the place, the food, and the actor. During the testing episode, since the actor was only associated with place $A$ in the habituation period, the babies predicted that the actor would go to place $A$ again, despite the food having been moved to place $B$. The babies did not necessarily have a mental picture of what the actor believed. As long as they memorized the last relevant event, then the reasoning sequence can be explained through CSO.

In a more sophisticated version of this experiment that sought to rule out the simple explanation (Onishi and Baillargeon 2005), the actor put a toy into a yellow box at location A during the habituation period. Then the position of the yellow box was swapped with that of an empty green box originally placed at location B while the view of the actor was blocked. When the actor returned, the baby was surprised if the actor looked for the toy in the green box at place A. In a second experiment, two green boxes were used in the same experiment. The babies were surprised if the actor looked for the toy in the green box at place B.

This new experiment seems to rule out the possibility that the babies can predict where the actor will look based on location association. However, the proposed CSO theory is still compatible with a simple explanation. In the case with the yellow box, the babies may have formed the concept of the yellow box during the habituation period and took the yellow color rather than its location as an associated concept with the toy. When the actor returned to the scene, the concept of the yellow box and the actor triggered the past event of their interactions and the location information might have been ignored. When two green boxes were used, the colors of the boxes were the same and may not have been picked up as a concept index for the event. Instead, location information was picked up as a concept just like in the original experiment. When the actor returned, the concept of the actor and the concept of location $A$ triggered a past event of their interactions at the same location. Since concepts are selectively generated and associated in different events, some information could have been ignored. Rich explanations are also compatible with the event-based reasoning theory with the additional mind-reading innate analyzers that track the actor's mind state. However, even with that capability, the babies would still face the choice of whether to associate the toy with the color of the box or with the location of the box, which will not change the basic mechanism of eventbased reasoning.

When young babies gradually develop concept mediated reasoning systems of CS1, CSO will be superseded. However when CS2 has not been fully developed, it is possible that babies display somewhat regressed cognitive ability due to the limitations of CS1. 
Indeed, the language development pattern supports this hypothesis. It is reported that children do not begin using complement structures with embedded false propositions although the overall sentence is right (De Villiers 2005). This indicates that children can start to imagine and compose longer events through DECM after age four.

Consider the scenario when only CS1 is used for reasoning. Since CS1 responds to activated conceptual nodes only through the DECM module, after the food was hidden at place $B$ and the actor entered the scene, the only activated conceptual nodes were those of food in place B and the actor, based on which, the DECM composed the event as "The actor will look for food at place B". This falls in line with the typical What You See Is All There Is (WYSIATI) characteristic of CS1, or the tendency to focus on existing evidence and ignore absent evidence. This behavior is very different from event-based reasoning in CSO.

An interesting phenomenon is that in these experiments, although toddlers between age 2 to 4 answered incorrectly, they reliably looked at the location where the agent last saw the food (Clements and Perner 1994). This indicates that both CSO and CS1 are at work at the same time, yet verbal communication prompted the toddlers to use CS1 reasoning to answer the question.

Later, as children mature, , they can employ CS2 or a combination of CSO and CS2 to construct an authentic theory-of-mind that allows them to predict correctly. Given that false-belief tasks require young children to imagine that the actor will look for food at hiding place $A$ after he saw food being hidden at place A, i.e. a complete event comparable in complexity to that of a complete sentence with an embedded statement, it is not surprising that they cannot accomplish false-belief tasks before age 4 .

This example indicates that the development process of DECM is reflected in children's language development, i.e., the ability of composing complete sentences reflects their ability to construct a complete episode of imagined events out of concepts. The gradual maturing of DECM explains the discontinuity in false-belief tasks capabilities. The fact that children's ability to accomplish false-belief tasks goes through three phases is additional evidence for the proposed three-tiered conceptual system theory.

\section{Section 6: The Objective-Subjective Perspective of the Conceptual System}

The issue of concern is that our concepts have great interpersonal and intra-person variabilities, which is indicative of the subjective nature of our conceptual system (Barsalou 1982, 1983). On the other hand, all past arguments for a completely subjective conceptual system have been unconvincing because it is tantamount to challenging the basis of scientific research, which requires objectivity. Also, if there were absolutely no objectivity in our conceptual system, then people from different cultural backgrounds would have very little chance to communicate and reach agreements. Thus, it is essential to understand the basis that spawns objectivity and subjectivity in the conceptual system. Given the opposing views on the subjective or objective nature of the conceptual system, two-tiered theories have been developed that try to reconcile the two opposing views. According to such theories, the narrow content of concepts are their subjective features and wide content their objective features (Pelletier 2017). Yet another 
theory considers features related to membership judgement to be objective and features related to typicality judgements to be subjective (Smith and Osherson 1984; Smith and Medin 1981). However, such theories lack a "nexus", or point of contact between the two tiers. Some scholars think that the nexus lies in lexical terms, in the sense that each word has an objective part corresponding to its lexical formulation and a subjective part when it is mentioned (Pelletier 2017).

Without a systematic account of how concepts are created and utilized in the conceptual system, it is impossible to understand its subjective nature and objective basis. In my threetiered theory, the innate analyzer outputs at the CSO level form the objective basis of the conceptual system; it is an automatic process that converts external stimuli to analog signals through innate analyzers. The subsequent concept-forming process introduces subjectivity because different features can be included in a concept in the abstraction process and the resulting feature space can be segmented in different ways depending on the different experiences of the subjects. However, given similar experiences, natural kinds/categories are largely determined in an automatic process by grouping natural clusters of features together, which varies little from subject to subject. Also, since CSO reasoning is based on real past events, beyond the problem of limited exposure, it is not biased by the subject's own conditions.

In CS1, since reasoning is conducted by DECM based on the composed events, it takes prototypes of activated conceptual nodes and the subject's emotional mood as its inputs. The level of subjectivity is the strongest because it is highly affected by the mood of the subject and the set of concepts last activated. Also, because prototypes and associative links in CS1 are formed by statistically averaging concept prototypes and their associations, CS1 is highly skewed to experiences (Kahneman 2011) . If a subject is limited in experience, then CS1 reasoning will be highly biased.

In CS2, since conceptual nodes can be formed by learning language alone and some can be aligned with primitive conceptual nodes, the basis is formed for communication within a community. Because the construction of the conceptual nodes is no longer mandated through the naturally existing clusters of concepts, they are largely a social construct, and they are "culturally subjective" because concepts will vary from culture to culture.

The flexible structure of CS2 enables logical reasoning, which aims at finding rules and laws that describe how one set of features of a category (of concepts) varies with another set of features. Through practical examination, the rules and laws can be validated based on observable phenomena. Subsequently, hypotheses proposed by DECM can be examined to reduce the error rate. However, nothing within the CS2 logical reasoning process can guarantee its absolute objectivity because the logical reasoning process requires subjective and selective focus on the relationships among a subset of features while assuming the rest of features are stable and irrelevant. 
Since CS1 is based on DECM, which is also the basis of our imagination, it introduces a large degree of subjectivity in our reasoning system. Information processing is more costly in CSO and CS2 because of their requirements to review previous events or to delve deep into the knowledge system. CS1 operation demands less mental energy so we unfortunately tend to stay in this subjective mood of thinking.

Subjectivity in our conceptual system can also be introduced by goals and desires, which have the effect of ruling out relevant events that do not fit into our interests. Consequently, even CSO and CS2 thinking cannot rule out subjectivity.

A better understanding of the source of our subjectivity will greatly enhance our ability to bridge differences between people. To become objective, we must broaden our experiences and minimize the influence of our desires and emotions.

\section{Section 7 Discussion}

I have shown that there must exist an event-mediated reasoning system prior to the establishment of CS1 and CS2, which is called Conceptual System 0 (CSO). In CS0, the major functional modules are innate analyzers, event memories, and primitive conceptual nodes linked to event memories. Besides these modules, there must also exist an event comparator that determines if a new event is interesting or not and a conceptual node generating mechanism which I did not discuss extensively in the paper. Nonetheless, they are integral and important parts of the structure that require future research. CSO forms the foundation of CS1 which further adds associative links and prototypes to conceptual nodes by statistically averaging events associated with the conceptual nodes. Associative reasoning in CS1 is achieved through a Dynamic Event Composition Module (DECM), which takes activated conceptual nodes and their associated conceptual nodes as inputs and conducts reasoning by composing likely events based on the inputs. In addition, I investigated the structural aspects of CS2 and identified pointers, variables, linked lists, relational conceptual nodes and knowledge systems as the critical components that make logical reasoning in CS2 possible. The significance of these findings is that they allow us to develop a parsimonious account of the acquisition, learning, and reasoning process that can best reconcile various puzzling phenomena observed in experimental studies.

The three-tiered system structure has also provided satisfactory answers to the three questions posed before: "Why does the meaning of concepts always change with contexts?", "Are most concepts learned or innate?", "Do various concept forms, such as definitions, exemplars, and prototypes, exist as multiple entities or are they associated with a core?"

Regarding the first question, in line with invariantism, this theory states that events associated with a concept give rise to its varying contextual meaning and the set of features identified during the learning phase of a concept give rise to its context-independent component. 
Furthermore in CS1, activated concepts are inputs to DECM, which composes likely events that provide meanings to concepts. Since the set of activated concepts and the emotional state of the subject are always different, concept meaning will always vary as stated by contextualism. Thus, the difference between invariantism and contextualism can be reconciled by realizing that they are actually describing characters of CSO and CS1, respectively.

Regarding the second question, given that primitive concepts are created from innate analyzer outputs through abstraction and segmentation steps, the theory acquires an innate aspect because objects will automatically be projected onto a point in the multidimensional space spanned by the innate analyzer outputs. Natural kinds will form natural clusters that can be separated from each other without a learning process. However, it also has a learning aspect that the subject has to be exposed to experiences before concepts can be abstracted and segmented. This reconciles the dispute between massive nativism and empiricism. In the past, innate analyzers were thought to output concepts directly, which created the disagreement between massive nativists and empiricists.

Regarding the third question, the proposed theory states that different forms of concepts are generated in different stages of concept development and they are employed in different processes for different purposes. After primitive conceptual nodes are formed through the abstraction and segmentation process at CSO, prototypes of concepts can be formed by statistically averaging feature values that satisfy the triggering conditions of the conceptual node. Subsequently, prototypes can be employed in concept identification and CS1 associative reasoning through DECM. On the other hand, CS2 allows the system to construct theories of concepts and employ them in CS2 reasoning. The attachment of prototypes, phonological and morphological symbols, as well as associative links among concepts, provide additional activation mechanisms for the underlying primitive conceptual node in addition to the triggering mechanism based on innate analyzer outputs. Due to the complexity in activating conceptual nodes, the set of evoked features will vary, which will cause significant variation in concept meaning.

One intriguing question is how exemplars are anchored in the proposed conceptual system framework. Past research defined them as instances of concepts stored in the long term memory (Nosofsky 1986, 1992; Medin and Schaffer 1978). They are invoked in concept categorization (Nosofsky 1986), reasoning (Juslin and Persson 2002; Hammond 1990) and concept compositions(Barsalou 2017; Hampton 1988). Many of these studies were conducted based on artificially constructed exemplars and they seem to imply that exemplars are standalone mental representations extracted from past experiences. In this paper, I take the view that exemplars are the referents of conceptual nodes embedded in past events. Exemplars are not stand-alone mental representations like prototypes and theories of concepts.

Consequently, exemplars are always invoked by contextual information. In contrast, prototypes and theories are context independent and only acquire their contextual meaning when they are used to compose concepts in CS1. 
Since primitive conceptual nodes and CS2 conceptual nodes are generated by completely different mechanisms, they exhibit completely different characteristics. Primitive conceptual nodes connected to episodic events and concepts like "dog" obtain their meaning from the set of events that involve a dog. Besides learning phase features, their meaning can be continuously learned and enriched by new experiences of events. In this sense, primitive concepts gain their informational semantics from their referents in the world.

In contrast, CS2 conceptual nodes are not directly linked to past events but are linked to other conceptual nodes. Consequently, they derive their meaning from their relationships to other concepts. For example, a bachelor is an unmarried man and the concepts of "unmarried" and " man" contribute to the meaning of "bachelor". In fact, the generation of such a CS2 conceptual node is an artifact. Except for convenience, there is no reason why we have to use this word.

Fodor had advocated for the idea that most primitive concepts derive their meaning from informational semantics. However, he failed to explain how a concept like "Tuesday" can be primitive (Fodor 1998). Given the proposed 3-tiered conceptual theory, we can see that "Tuesday" derives its meaning entirely based on its position in a linked list and the dates it points to. The arguments between informational semantics and inferential role semantics can be resolved by realizing that concepts like "dog", "bachelor", and "Tuesday" are formed based on entirely different mechanisms situated in different tiers of the conceptual system.

As a central part of my conceptual system theory, I argue that prelinguistic infants and animals rely on event-based reasoning to make inferences of what will happen given past or current information in CSO. CSO enables a fast learning process because even a single encounter of an event can be used for later reasoning. However CSO is not efficient for fast decision making because it needs to go over all relevant past events to compute the best match. CS1 expedites the reasoning process by making associations between concepts that frequently co-occur in experience and making predictions through DECM based on activated concepts. This composing mechanism utilizes prototypes of concepts and is affected by the physiological and emotional state of the subject. The host of observed phenomena observed in System 1 reasoning (Kahneman 2011) can be explained by the existence of such a mechanism. With the introduction of DECM, CS1 reasoning assumes completely different characteristics from that of CSO because it introduces imagination as well as subjectivity into the reasoning process.

With the emergence of human language, conceptual nodes not directly linked to innate analyzer outputs can be created with great flexibility in CS2, because they can be linked to other conceptual nodes. CS2 is centered around the capability of finding rules/laws and applying them. The central functions are entailed in RFCM, which still employs DECM; however, the number of activated concepts can be significantly expanded in CS2 due to its larger knowledge system and consequent reduction in prediction error. Furthermore, composed events can be examined by the rules stored in the knowledge system to reduce error rate. The reasoning process can be conducted by rejecting unlikely hypotheses generated by DECM in an iterative process. 
Due to the abstraction and segmentation steps in concept generation, limitations exist in logical reasoning. The conceptual ambiguities embedded in the puzzling phenomena of Kant's antinomies have been analyzed.

Much of the research on CS2 is still needed to understand the detailed mechanism. For example, besides conscious thinking, is there an intuitive process that directly finds rules based on CSO events and generates CS2 rules? Is RFCM automatically or consciously utilized? I take the view that the function of RFCM is partially automatic, which can explain the intuitive discoveries of scientific theories.

The proposed conceptual system theory answers many questions and opens many questions for future research. We can further explore from a philosophical perspective that cannot be replaced by experimental-based research that focuses on probing new problems and accumulating new observations. Philosophical research, on the other hand, focuses on establishing new concepts based on all experiences. What has not been included in the paper is the impact of such a theory on traditional topics of philosophy. For example, the exploration of the theory's impact on cosmological, ontological, teleological views in metaphysics and the applicability of logical reasoning in various cases.

Another area that can be further explored is the research on creativity. For example, one type of creativity is related to the new combinations of existing concepts through DECM. Another type of creativity is related to the development of new concepts based on existing events. Yet another type of creativity involves how to recognize new rules in CS2. For these various types of creativity, the implied mind states are also different. For example, if we want to grow new conceptual nodes from existing events, we need to make sure that existing conceptual nodes are not stimulated. Since existing concepts are triggered by either reading, listening, thinking, or perceiving through sensory organs, does it imply that growing new conceptual nodes from existing events requires us to shut out all these interferences? Besides not triggering existing concepts, what other conditions are required to initiate the process of growing new conceptual nodes? Once future research clarifies these questions, it will help us to gain more insight into how we create new concepts.

In artificial intelligence, we can apply some of the insights to design more efficient and more intelligent machines. For example, for the categorization of unknown objects, past research mostly employed supervised and unsupervised learning based on static images but not episodic events. If the input of the learning system is event based, we might be able to design artificial intelligence that is capable of reasoning in different contexts.

In the social science area, we can investigate how the conceptual system differs from one group of people to another, which is a source of conflicts between individuals and between cultures. How can we bridge the differences in a scientific and efficient way and how can we eliminate these gaps in perception is one of the most important challenges facing this era. 
Lastly and most importantly, we need to address the ethical and safety concerns in developing artificial intelligence, especially given that large scale data mining systems like the Bert system train neural networks with billions of parameters, which have built a formidable knowledge database capable of CS1 and partial CS2 reasoning without a clear understanding of the implications. If we further add creativity to artificial intelligence, whether intentionally or accidentally, the unlimited computing power, would make it extremely dangerous. Thus, based on a deeper understanding, we must further the research on safe artificial intelligence control protocols.

The understanding of the conceptual system is at the core of all sciences, and will bring significant progress in numerous areas.

Barner, David, Dora Thalwitz, Justin Wood, Shu-Ju Yang, and Susan Carey. 2007. "On the Relation between the Acquisition of Singular--Plural Morpho-Syntax and the Conceptual Distinction between One and More than One." Developmental Science 10 (3): 365-73.

Barner, David, Justin Wood, Marc Hauser, and Susan Carey. 2008. "Evidence for a NonLinguistic Distinction between Singular and Plural Sets in Rhesus Monkeys." Cognition 107 (2): 603-22.

Baroni, Marco, and Alessandro Lenci. 2010. "Distributional Memory: A General Framework for Corpus-Based Semantics.” Computational Linguistics 36 (4): 673-721.

Barsalou, Lawrence. 1987. "The Instability of Graded Structure: Implications for the Nature of Concepts." In Concepts and Conceptual Development: Ecological and Intellectual Factors in Categorization, edited by U. Neisser, 101-40. Cambridge University Press.

Barsalou, Lawrence W. 1991. "Deriving Categories to Achieve Goals." In Psychology of Learning and Motivation, edited by Gordon H. Bower, 27:1-64. Academic Press.

- 2008. "Grounded Cognition." Annual Review of Psychology. https://doi.org/10.1146/annurev.psych.59.103006.093639.

- 2017. "Cognitively Plausible Theories of Concept Composition." In Compositionality and Concepts in Linguistics and Psychology, 9-30. Springer, Cham.

Barsalou, Lawrence W., Ava Santos, W. Kyle Simmons, and Christine D. Wilson. 2008. "Language and Simulation in Conceptual Processing." Symbols and EmbodimentDebates on Meaning and Cognition. https://doi.org/10.1093/acprof:oso/9780199217274.003.0013.

Barsalou, L. W. 1982. "Context-Independent and Context-Dependent Information in Concepts." Memory \& Cognition 10 (1): 82-93.

—. 1983. "Ad Hoc Categories.” Memory \& Cognition 11 (3): 211-27. . 1999. "Perceptual Symbol Systems." The Behavioral and Brain Sciences 22 (4): 577609; discussion 610-60.

Berger, Jonah, Marc Meredith, and S. Christian Wheeler. 2008. "Contextual Priming: Where People Vote Affects How They Vote." Proceedings of the National Academy of Sciences of the United States of America 105 (26): 8846-49.

Block, Ned. 1986. "Advertisement for a Semantics for Psychology.” Midwest Studies in Philosophy 10 (April): 615-78.

Block, Ned, and John Campell. 1987. "Functional Role and Truth Conditions." Proceedings of the Aristotelian Society, Supplementary Volumes 61: 157-83.

Bolte, Annette, Thomas Goschke, and Julius Kuhl. 2003. "Emotion and Intuition: Effects of 
Positive and Negative Mood on Implicit Judgments of Semantic Coherence." Psychological Science 14 (5): 416-21.

Boodin, John Elof. 1939. "The Social Mind: Foundations of Social Philosophy." https://doi.org/10.1037/14683-000.

Boyd, Richard. 1999. "Homeostasis, Species, and Higher Taxa." Species: New Interdisciplinary Essays 141: 185.

Brannon, Elizabeth M. 2002. "The Development of Ordinal Numerical Knowledge in Infancy." Cognition 83 (3): 223-40.

Brannon, Elizabeth M., Sara Abbott, and Donna J. Lutz. 2004. "Number Bias for the Discrimination of Large Visual Sets in Infancy." Cognition 93 (2): B59-68.

Brannon, Elizabeth M., and Herbert S. Terrace. 1998. "Ordering of the Numerosities 1 to 9 by Monkeys." Science 282 (5389): 746-49.

Brigandt, Ingo. 2009. "Natural Kinds in Evolution and Systematics: Metaphysical and Epistemological Considerations." Acta Biotheoretica 57 (1-2): 77-97.

Brooks, Lee R. 1978. "Nonanalytic Concept Formation and Memory for Instances." https://philarchive.org/rec/BRONCF.

Carey, Susan. 1985. Conceptual Change in Childhood. MIT press.

- 1999. "Sources of Conceptual Change." Conceptual Development: Piaget's Legacy, 293-326.

- 2000. "The Origin of Concepts." Journal of Cognition and Development: Official Journal of the Cognitive Development Society 1 (1): 37-41.

- 2014. "On Learning New Primitives in the Language of Thought: Reply to Rey." Mind \& Language 29 (2): 133-66.

- 2015. "Why Theories of Concepts Should Not Ignore the Problem of Acquisition." Disputatio 7 (41): 113-63.

Carey, Susan, and Elizabeth Spelke. 1994. "Domain-Specific Knowledge and Conceptual Change." Mapping the Mind: Domain Specificity in Cognition and Culture 169: 200.

Caruso, Eugene M., Oren Shapira, and Justin F. Landy. 2017. "Show Me the Money: A Systematic Exploration of Manipulations, Moderators, and Mechanisms of Priming Effects." Psychological Science 28 (8): 1148-59.

Casasanto, Daniel, and Gary Lupyan. 2015. "All Concepts Are Ad Hoc Concepts.” The Conceptual Mind: New Directions in the Study of Concepts 543: 566.

Chang, Le, and Doris Y. Tsao. 2017. "The Code for Facial Identity in the Primate Brain." Cell 169 (6): 1013-28.e14.

Clements, Wendy A., and Josef Perner. 1994. "Implicit Understanding of Belief." Cognitive Development 9 (4): 377-95.

Dalen, Dirk van. 2004. Logic and Structure. Springer, Berlin, Heidelberg.

Damasio, A. R. 1989. “Time-Locked Multiregional Retroactivation: A Systems-Level Proposal for the Neural Substrates of Recall and Recognition." Cognition 33 (1-2): 25-62.

De Villiers, Jill G. 2005. "IQ Can Language Acquisition Give Children a Point ofView." Why Language Matters for Theory of Mind 186.

https://books.google.com/books?hl=en\&lr=\&id=NWDs-

aHKodsC\&oi $=$ fnd \&pg $=$ PA186\&dq $=$ can + language + acquisition + give $\&$ ots $=38 \mathrm{NxeXxP0y \& s}$ ig=EZ4gUbSGuo-sXvG-9nyEac9ffhc.

Devlin, Jacob, Ming-Wei Chang, Kenton Lee, and Kristina Toutanova. 2018. "BERT: PreTraining of Deep Bidirectional Transformers for Language Understanding." arXiv [cs.CL]. 
arXiv. http://arxiv.org/abs/1810.04805.

Emlen, S. T. 1975. "The Stellar-Orientation System of a Migratory Bird." Scientific American 233 (2): 102-11.

Erk, Katrin. 2012. "Vector Space Models of Word Meaning and Phrase Meaning: A Survey." Language and Linguistics Compass 6 (10): 635-53.

Evans, Jonathan St B. T. 2003. "In Two Minds: Dual-Process Accounts of Reasoning." Trends in Cognitive Sciences 7 (10): 454-59.

—. 2008. "Dual-Processing Accounts of Reasoning, Judgment, and Social Cognition." Annual Review of Psychology 59: 255-78.

Evans, Jonathan St B. T., and Keith Frankish. 2009. In Two Minds: Dual Processes and beyond. Vol. 10. Oxford University Press Oxford.

Evans, Vyvyan. 2009. How Words Mean: Lexical Concepts, Cognitive Models, and Meaning Construction. OUP Oxford.

- 2015. "10 What's in a Concept? Analog versus Parametric Concepts in LCCM Theory." The Conceptual Mind: New Directions in the Study of Concepts, 251.

Fodor, Jerry A. 1975. The Language of Thought. Harvard University Press.

-1981. "The Present Status of the Innateness Controversy." https://philarchive.org/rec/FODTPS.

- 1983. The Modularity of Mind. MIT Press.

-1998. Concepts: Where Cognitive Science Went Wrong. Oxford University Press.

—. 2001. "Language, Thought and Compositionality." Mind and Language. https://doi.org/10.1111/1468-0017.00153.

- 2008. LOT 2: The Language of Thought Revisited. OUP Oxford.

Fodor, Jerry A., and Ernest LePore. 2002. The Compositionality Papers. Clarendon Press.

Fodor, J., and E. Lepore. 1996. "The Red Herring and the Pet Fish: Why Concepts Still Can't Be Prototypes." Cognition 58 (2): 253-70.

Francez, Nissim. 2015. Proof-Theoretic Semantics. College Publications London.

Gao, Huiying, and Xiuxiu Chen. 2012. "Ontology and CBR-Based Dynamic Enterprise Knowledge Repository Construction.” JSW 7 (6): 1211-18.

Glaser, W. R. 1992. "Picture Naming." Cognition 42 (1-3): 61-105.

Gleitman, Lila. 1990. "The Structural Sources of Verb Meanings.” Language Acquisition 1 (1): $3-55$.

Goddard, Cliff, and Anna Wierzbicka. 1994. Semantic and Lexical Universals: Theory and Empirical Findings. John Benjamins Publishing.

- 2002. "2. Semantic Primes and Universal Grammar." Studies in Language Companion Series. https://doi.org/10.1075/slcs.60.08god.

Gopnik, Alison, Clark Glymour, David M. Sobel, Laura E. Schulz, Tamar Kushnir, and David Danks. 2004. "A Theory of Causal Learning in Children: Causal Maps and Bayes Nets." Psychological Review. https://doi.org/10.1037/0033-295x.111.1.3.

Grandy, Richard E. 1990. "Understanding and the Principle of Compositionality." Philosophical Perspectives. A Supplement to Nous 4: 557-72.

Hammond, Kristian J. 1990. "Case-Based Planning: A Framework for Planning from Experience." Cognitive Science 14 (3): 385-443.

- 2012. Case-Based Planning: Viewing Planning as a Memory Task. Elsevier.

Hampton, J. A. 1987. "Inheritance of Attributes in Natural Concept Conjunctions." Memory \& Cognition 15: 55-71. 
Hampton, James A. 1988. "Overextension of Conjunctive Concepts: Evidence for a Unitary Model of Concept Typicality and Class Inclusion." Journal of Experimental Psychology. Learning, Memory, and Cognition 14 (1): 12-32.

. 1979. "Polymorphous Concepts in Semantic Memory." Journal of Verbal Learning and Verbal Behavior 18 (4): 441-61.

—. 1997. "Conceptual Combination." Knowledge, Concepts, and Categories, 133-59. 2017. "Compositionality and Concepts." In Compositionality and Concepts in Linguistics and Psychology, 95-121. Springer, Cham.

Hegel, Georg Wilhelm Friedrich. 1929. "Hegel's Science of Logic." https://philarchive.org/rec/HEGHSO.

Hoenig, Klaus, Eun-Jin Sim, Viktor Bochev, Bärbel Herrnberger, and Markus Kiefer. 2008. "Conceptual Flexibility in the Human Brain: Dynamic Recruitment of Semantic Maps from Visual, Motor, and Motion-Related Areas." Journal of Cognitive Neuroscience 20 (10): 1799-1814.

Holdstock, J. S., A. R. Mayes, N. Roberts, E. Cezayirli, C. L. Isaac, R. C. O'Reilly, and K. A. Norman. 2002. "Under What Conditions Is Recognition Spared Relative to Recall after Selective Hippocampal Damage in Humans?" Hippocampus 12 (3): 341-51.

Hsu, Jeremy. 2008. "The Secrets of Storytelling: Why We Love a Good Yarn." Scientific American Mind 19 (4): 46-51.

Huttenlocher, Janellen, and Larry V. Hedges. 1994. "Combining Graded Categories: Membership and Typicality." Psychological Review. https://doi.org/10.1037/0033295x.101.1.157.

Jackendoff, Ray. 1992. Semantic Structures. MIT Press.

James, Thomas W., and Isabel Gauthier. 2003. "Auditory and Action Semantic Features Activate Sensory-Specific Perceptual Brain Regions.” Current Biology. https://doi.org/10.1016/j.cub.2003.09.039.

-J. Ch. Meyer, J., and W. van der Hoek. 2004. Epistemic Logic for AI and Computer Science. Cambridge University Press.

Juslin, Peter, and Magnus Persson. 2002. "PROBabilities from EXemplars (PROBEX): A 'lazy' Algorithm for Probabilistic Inference from Generic Knowledge." Cognitive Science 26 (5): 563-607.

Kahneman, Daniel. 2011. Thinking, Fast and Slow. Macmillan.

Kahneman, Daniel, and Dale T. Miller. 1986. "Norm Theory: Comparing Reality to Its Alternatives." Psychological Review 93 (2): 136-53.

Kant, Immanuel. 2002. Critique of Practical Reason. Hackett Publishing.

Keane, Margaret. 1999. "Combining Prototypes: A Selective Modification Model Edward E. Smith, Daniel N. Osherson, Lance J. Rips, and.” Concepts: Core Readings, 355.

Keil, Frank C., and Robert A. Wilson, eds. 2000. "Explanatory Understanding and Conceptual Combination.” In Explanation and Cognition. The MIT Press.

Kriegeskorte, Nikolaus, Marieke Mur, Douglas A. Ruff, Roozbeh Kiani, Jerzy Bodurka, Hossein Esteky, Keiji Tanaka, and Peter A. Bandettini. 2008. "Matching Categorical Object Representations in Inferior Temporal Cortex of Man and Monkey." Neuron 60 (6): 112641.

Kripkie, S. 1980. "Naming and Necessity." Blackwell. http://www.kaleybradley.com/McDaniel/courses/spring_2012/MandM/readings/Kripke-NandN.PDF.

Kumaran, Dharshan, Demis Hassabis, and James L. McClelland. 2016. "What Learning Systems 
Do Intelligent Agents Need? Complementary Learning Systems Theory Updated." Trends in Cognitive Sciences 20 (7): 512-34.

Kunda, Ziva, Dale T. Miller, and Theresa Claire. 1990. "Combining Social Concepts: The Role of Causal Reasoning." Cognitive Science 14 (4): 551-77.

Landauer, Thomas K., and Susan T. Dumais. 1997. "A Solution to Plato's Problem: The Latent Semantic Analysis Theory of Acquisition, Induction, and Representation of Knowledge." Psychological Review 104 (2): 211-40.

Laurence, Stephen, and Eric Margolis. 1999. "Concepts and Cognitive Science.” Concepts: Core Readings 3: 81.

- 2002. "Radical Concept Nativism." Cognition 86 (1): 25-55.

. 2015. "Concept Nativism and Neural Plasticity." The Conceptual Mind. New Directions in the Study of Concepts, 117-47.

LeCun, Yann, Yoshua Bengio, and Geoffrey Hinton. 2015. “Deep Learning.” Nature 521 (7553): 436-44.

Louwerse, Max, and Louise Connell. 2011. "A Taste of Words: Linguistic Context and Perceptual Simulation Predict the Modality of Words." Cognitive Science 35 (2): 381-98.

Machery, Edouard. 2009. Doing without Concepts. Oxford University Press.

- 2015. "By Default." The Conceptual Mind: New Directions in the Study of Concepts, $567-88$.

Macnamara, John. 1986. A Border Dispute: The Place of Logic in Psychology. Cambridge, Mass.: MIT Press.

Malt, Barbara C. 2010. "Why We Should Do Without Concepts.” Mind \& Language. https://doi.org/10.1111/j.1468-0017.2010.01405.x.

Margolis, Eric, and Stephen Laurence. 2011. "Learning Matters: The Role of Learning in Concept Acquisition." Mind \& Language 26 (5): 507-39.

Mayes, Andrew, Daniela Montaldi, and Ellen Migo. 2007. "Associative Memory and the Medial Temporal Lobes.” Trends in Cognitive Sciences 11 (3): 126-35.

McClelland, James L., Bruce L. McNaughton, and Andrew K. Lampinen. 2020. "Integration of New Information in Memory: New Insights from a Complementary Learning Systems Perspective." Cold Spring Harbor Laboratory. https://doi.org/10.1101/2020.01.17.909804.

McCrink, Koleen, and Karen Wynn. 2007. "Ratio Abstraction by 6-Month-Old Infants." Psychological Science 18 (8): 740-45.

Medin, D. L., and E. J. Shoben. 1988. "Context and Structure in Conceptual Combination." Cognitive Psychology 20 (2): 158-90.

Medin, Douglas L., and Marguerite M. Schaffer. 1978. "Context Theory of Classification Learning." Psychological Review 85 (3): 207-38.

—. 2017. "Category Learning Task.” PsycTESTS Dataset. https://doi.org/10.1037/t62895000.

Mednick, S. A. 1962. "The Associative Basis of the Creative Process." Psychological Review 69 (May): 220-32.

Michotte, Albert. 1946. "La Perception de La Causalité [The Perception of Causality].” Louvain: Institut Supérieur de Philosophie.

- 2017. The Perception of Causality. Routledge.

Murphy, G. L., and D. L. Medin. 1985. "The Role of Theories in Conceptual Coherence."

Psychological Review 92 (3): 289-316.

Murphy, Gregory L. 1988. “Comprehending Complex Concepts.” Cognitive Science 12 (4): 
$529-62$.

. 1990. "Noun Phrase Interpretation and Conceptual Combination." Journal of Memory and Language 29 (3): 259-88.

Nersessian, Nancy J. 1992. "How Do Scientists Think? Capturing the Dynamics of Conceptual Change in Science." Cognitive Models of Science 15: 3-44.

Nosofsky, Robert M. 1986. "Attention, Similarity, and the Identification-categorization Relationship.” Journal of Experimental Psychology. General 115 (1): 39-57.

—. 1992. "Exemplar-Based Approach to Relating Categorization, Identification, and Recognition." Multidimensional Models of Perception and Cognition. 523: 363-93.

Onishi, Kristine H., and Renée Baillargeon. 2005. "Do 15-Month-Old Infants Understand False Beliefs?" Science 308 (5719): 255-58.

Oppenheimer, Daniel M. 2006. "Consequences of Erudite Vernacular Utilized Irrespective of Necessity: Problems with Using Long Words Needlessly." Applied Cognitive Psychology 20 (2): 139-56.

Osherson, Daniel N., and Edward E. Smith. 1981. "On the Adequacy of Prototype Theory as a Theory of Concepts." Cognition 9 (1): 35-58.

Pagin, Peter. 2009. "Compositionality, Understanding, and Proofs." Mind; a Quarterly Review of Psychology and Philosophy 118 (471): 713-37.

- 2012. "Communication and the Complexity of Semantics," 510-29.

Papafragou, Anna. 2015. "12 The Representation of Events in Language and Cognition." The Conceptual Mind: New Directions in the Study of Concepts, 327.

Pearl, Judea. 2000. Causality: Models, Reasoning, and Inference. Cambridge University Press. Pelletier, Francis Jeffry. 2017. "Compositionality and concepts-A Perspective from Formal Semantics and Philosophy of Language." In Compositionality and Concepts in Linguistics and Psychology, 31-94. Springer, Cham.

Pepperberg, Irene M., and Susan Carey. 2012. "Grey Parrot Number Acquisition: The Inference of Cardinal Value from Ordinal Position on the Numeral List." Cognition 125 (2): 219-32.

Prinz, Jesse J. 2005. "The Return of Concept Empiricism.” In Handbook of Categorization in Cognitive Science, 679-95. Elsevier.

Putnam, Hilary. 1975. "The Meaning of "meaning." Philosophical Papers 2. https://books.google.com/books?hl=en\&lr=\&id=6YMYDQAAQBAJ\&oi=fnd\&pg=PT24\& $\mathrm{dq}=$ the + meaning + of + meaning + putnam\&ots $=\mathrm{J} \_$uFy2swMQ\&sig $=48$ tsbMGtYrGOSCZBpG $\mathrm{hV} \_$uFeZP8.

Pylyshyn, Z. W. 2001. "Visual Indexes, Preconceptual Objects, and Situated Vision." Cognition 80 (1-2): 127-58.

Pylyshyn, Z. W., and R. W. Storm. 1988. "Tracking Multiple Independent Targets: Evidence for a Parallel Tracking Mechanism.” Spatial Vision 3 (3): 179-97.

Quine, Willard Van Orman. 1960. "Word and Object. New Edition." Cambridge, Massachusetts: The. https://patriciachurchland.com/wp-content/uploads/2020/05/2013-Foreward-Wordand-Object.pdf.

Quine, W. O. 1977. "Natural kinds/Naming, Necessity, and Natural Kinds. Ithaca.” L.

Quine, W. V. 1974. The Roots of Reference. Lasalle, Ill., Open Court.

Rey, Georges. 2014. "Innate and Learned: Carey, Mad Dog Nativism, and the Poverty of Stimuli and Analogies (Yet Again) : Innate and Learned." Mind \& Language 29 (2): 109-32.

Rosch, Eleanor, and Carolyn B. Mervis. 1975. "Family Resemblances: Studies in the Internal Structure of Categories." Cognitive Psychology 7 (4): 573-605. 
Saxe, Rebecca, Tania Tzelnic, and Susan Carey. 2007. "Knowing Who Dunnit: Infants Identify the Causal Agent in an Unseen Causal Interaction." Developmental Psychology 43 (1): 14958.

Saxe, R., J. B. Tenenbaum, and S. Carey. 2005. "Secret Agents: Inferences About Hidden Causes by 10- and 12-Month-Old Infants." Psychological Science. https://doi.org/10.1111/j.1467-9280.2005.01649.x.

Schlottmann, A., and D. R. Shanks. 1992. "Evidence for a Distinction between Judged and Perceived Causality." The Quarterly Journal of Experimental Psychology. A, Human Experimental Psychology 44 (2): 321-42.

Sloman, Steven A. (1996). "The Empirical Case for Two Systems of Reasoning." Psychological Bulletin 119 (1): 3-22.

Smith, Edward E., and Douglas L. Medin. 1981. Categories and Concepts. Vol. 9. Harvard University Press Cambridge, MA.

Smith, Edward E., and Daniel N. Osherson. 1984. "Conceptual Combination with Prototype Concepts.” Cognitive Science 8 (4): 337-61.

Smith, Linda B., and Larissa K. Samuelson. 1997. "Perceiving and Remembering: Category Stability, Variability and Development." Knowledge, Concepts and Categories. 464: 16195.

Stanovich, K. E., and R. F. West. 2000. "Individual Differences in Reasoning: Implications for the Rationality Debate?" The Behavioral and Brain Sciences 23 (5): 645-65; discussion $665-726$.

Suraperwata, Raihan Hamid, and Suyanto Suyanto. 2020. "Language Modeling for Journalistic Robot Based on Generative Pretrained Transformer 2." 2020 8th International Conference on Information and Communication Technology (ICoICT). https://doi.org/10.1109/icoict49345.2020.9166359.

Surian, Luca, Stefania Caldi, and Dan Sperber. 2007. "Attribution of Beliefs by 13-Month-Old Infants." Psychological Science 18 (7): 580-86.

Teyler, T. J., and P. DiScenna. 1986. "The Hippocampal Memory Indexing Theory." Behavioral Neuroscience 100 (2): 147-54.

Tonegawa, Susumu, Mark D. Morrissey, and Takashi Kitamura. 2018. "The Role of Engram Cells in the Systems Consolidation of Memory." Nature Reviews. Neuroscience 19 (8): 485-98.

Tooby, John, and Leda Cosmides. 2015. "Conceptual Foundations of Evolutionary Psychology." The Handbook of Evolutionary Psychology. https://doi.org/10.1002/9780470939376.ch1.

Tse, Dorothy, Rosamund F. Langston, Masaki Kakeyama, Ingrid Bethus, Patrick A. Spooner, Emma R. Wood, Menno P. Witter, and Richard G. M. Morris. 2007. "Schemas and Memory Consolidation." Science 316 (5821): 76-82.

Tse, Dorothy, Tomonori Takeuchi, Masaki Kakeyama, Yasushi Kajii, Hiroyuki Okuno, Chiharu Tohyama, Haruhiko Bito, and Richard G. M. Morris. 2011. "Schema-Dependent Gene Activation and Memory Encoding in Neocortex." Science 333 (6044): 891-95.

Tulving, Endel, Daniel L. Schacter, and Heather A. Stark. 1982. "Priming Effects in WordFragment Completion Are Independent of Recognition Memory." Journal of Experimental Psychology. Learning, Memory, and Cognition 8 (4): 336-42.

Tulving, Endel, and Donald M. Thomson. 1973. "Encoding Specificity and Retrieval Processes in Episodic Memory." Psychological Review 80 (5): 352-73.

Van Orman Quine, Willard. 1969. Ontological Relativity and Other Essays. Columbia 
University Press.

Verhage, M., A. S. Maia, J. J. Plomp, A. B. Brussaard, J. H. Heeroma, H. Vermeer, R. F. Toonen, et al. 2000. "Synaptic Assembly of the Brain in the Absence of Neurotransmitter Secretion." Science 287 (5454): 864-69.

Vohs, Kathleen D., Nicole L. Mead, and Miranda R. Goode. 2006. "The Psychological Consequences of Money." Science 314 (5802): 1154-56.

Weiskopf, Daniel. 2015. "Observational Concepts." The Conceptual Mind: New Directions in the Study of Concepts, 223-47.

Wellman, Henry M., David Cross, and Julanne Watson. 2001. "Meta-Analysis of Theory-ofMind Development: The Truth about False Belief." Child Development 72 (3): 655-84.

Wierzbicka, Anna. 2015. "14 Innate Conceptual Primitives Manifested in the Languages of the World and in Infant Cognition." The Conceptual Mind: New Directions in the Study of Concepts, 379.

Wierzbicka, Anna, and Cliff Goddard. 2014. "Words and Meanings: Lexical Semantics across Domains, Languages and Cultures." Oxford: Oxford University Press.

Wimmer, H., and J. Perner. 1983. "Beliefs about Beliefs: Representation and Constraining Function of Wrong Beliefs in Young Children's Understanding of Deception." Cognition 13 (1): 103-28.

Wu, Ling-Ling, and Lawrence W. Barsalou. 2009. "Perceptual Simulation in Conceptual Combination: Evidence from Property Generation." Acta Psychologica 132 (2): 173-89.

Xu, Fei, Elizabeth S. Spelke, and Sydney Goddard. 2005. "Number Sense in Human Infants." Developmental Science 8 (1): 88-101.

Xu, F., and E. S. Spelke. 2000. "Large Number Discrimination in 6-Month-Old Infants." Cognition 74 (1): B1-11.

Zacks, J. M., and B. Tversky. 2001. "Event Structure in Perception and Conception." Psychological Bulletin 127 (1): 3-21.

Zajonc, R. B. 2001. "Mere Exposure: A Gateway to the Subliminal." Current Directions in Psychological Science 10 (6): 224-28. 